\title{
Mass motions and chromospheres of RGB stars in the globular cluster NGC 2808*
}

\author{
C. Cacciari ${ }^{1}$, A. Bragaglia ${ }^{1}$, E. Rossetti ${ }^{1}$, F. Fusi Pecci $^{1}$, G. Mulas ${ }^{2}$, \\ E. Carretta ${ }^{3}$, R. G. Gratton ${ }^{3}$, Y. Momany ${ }^{4}$, and L. Pasquini ${ }^{5}$ \\ 1 INAF-Osservatorio Astronomico di Bologna, via Ranzani 1, 40126 Bologna, Italy \\ e-mail: cacciari@bo.astro.it; angela@bo.astro.it; emanuel@bo.astro.it; flavio@bo.astro.it \\ 2 INAF-Osservatorio Astronomico di Cagliari, Loc. Poggio dei Pini, Strada 54, 09012 Capoterra (CA), Italy \\ e-mail: gmulas@ca.astro.it \\ 3 INAF-Osservatorio Astronomico di Padova, vicolo Osservatorio 5, 35122 Padova, Italy \\ e-mail: carretta@pd.astro.it; gratton@pd.astro.it \\ 4 Università di Padova, Dip. di Astronomia, vicolo Osservatorio 2, 35122 Padova, Italy \\ e-mail: momany@pd.astro.it \\ 5 European Southern Observatory, Karl-Schwarzschild-Str. 2, 85749 Garching b. München, Germany \\ e-mail: lpasquin@eso.org
}

Received 19 June 2003 / Accepted 22 September 2003

\begin{abstract}
We present the results of the first observations, taken with FLAMES during Science Verification, of red giant branch (RGB) stars in the globular cluster NGC 2808. A total of 137 stars was observed, of which 20 at high resolution $(R=47000)$ with UVES and the others at lower resolution $(R=19000-29000)$ with GIRAFFE in MEDUSA mode, monitoring $\sim 3$ mag down from the RGB tip. Spectra were taken of the $\mathrm{H} \alpha$, Na I D and Ca II H and K lines. This is by far the largest and most complete collection of such data in globular cluster giants, both for the number of stars observed within one cluster, and for monitoring all the most important optical diagnostics of chromospheric activity/mass motions. Evidence of mass motions in the atmospheres was searched from asymmetry in the profiles and coreshifts of the $\mathrm{H} \alpha$, Na I D and Ca II K lines, as well as from $\mathrm{H} \alpha$ emission wings. We have set the detection thresholds for the onset of $\mathrm{H} \alpha$ emission, negative Na $D_{2}$ coreshifts and negative $K_{3}$ coreshifts at $\log L / L_{\odot} \sim 2.5,2.9$ and 2.8, respectively. These limits are significantly fainter than the results found by nearly all previous studies. Also the fraction of stars where these features have been detected has been increased significantly with respect to the previous studies. Our observations confirm the widespread presence of chromospheres among globular cluster giants, as it was found among Population I red giants. Some of the above diagnostics suggest clearly the presence of outward mass motions in the atmosphere of several stars.
\end{abstract}

Key words. line: profiles - globular clusters: individual: NGC 2808 - stars: atmospheres - stars: mass loss stars: Population II - techniques: spectroscopic

\section{Introduction}

One of the most solid requirements of the stellar evolution theory is that some amount of mass loss (a few tenths of a solar mass) must occur during the evolutionary phases preceding the Horizontal Branch (HB) phase, in order to account for the observed HB morphologies in globular clusters (GC) (Castellani \& Renzini 1968; Iben \& Rood 1970; Rood 1973; Fusi Pecci \& Renzini 1975, 1976; Renzini 1977). Also the pulsational properties of the RR Lyrae variables and the absence of asymptotic giant branch (AGB) stars brighter than the red giant branch (RGB) tip require that some mass has

Send offprint requests to: C. Cacciari,

e-mail: cacciari@bo.astro.it

* Based on observations collected at the European Southern Observatory, Chile, during FLAMES Science Verification. been lost during previous evolutionary phases (Christy 1966; Fusi Pecci et al. 1993; D'Cruz et al. 1996). Theoretical estimates of mass loss rates at the tip of the RGB are a few times $10^{-8} M_{\odot} \mathrm{yr}^{-1}$ (Fusi Pecci \& Renzini 1975, 1976; Renzini 1977). This would produce a few tens of solar masses of intracluster matter that should accumulate in the central regions of the clusters, in absence of sweeping mechanisms between Galactic plane crossings.

However, efforts to obtain direct evidence of intracluster matter, or of mass loss from individual RGB stars, have been only marginally successful. Diffuse gas in GC's was detected only as an upper limit and well below $1 M_{\odot}$ (Roberts 1988; Smith et al. 1990; Faulkner \& Smith 1991; Freire et al. 2001), whereas the most recent search of mass loss evidence from individual RGB (or AGB) stars via ISOCAM IR-excess has indeed shown that dusty circumstellar envelopes are present 
in $\sim 15 \%$ of the giants in the $\sim 0.7$ mag brightest interval $\left(M_{\mathrm{bol}} \leq\right.$ -2.5) (Origlia et al. 2002, and references therein).

Spectroscopic surveys of a few hundred GC red giants (Cohen 1976, 1978, 1979, 1980, 1981; Mallia \& Pagel 1978; Peterson 1981, 1982; Cacciari \& Freeman 1983; Gratton et al. 1984) did reveal $\mathrm{H} \alpha$ emission wings in a good fraction of stars brighter than $\log L / L_{\odot} \sim 2.7$, i.e. along the upper $1.25 \mathrm{mag}$ interval of the RGB. This was initially interpreted as evidence of an extended atmosphere, i.e. of mass loss. However, Dupree et al. (1984) demonstrated that this emission per se is not a unambiguous mass loss indicator, as it could arise naturally in a static stellar chromosphere, or it could be influenced by hydrodynamic processes due to pulsation (Dupree et al. 1994).

Profile asymmetry and coreshifts of chromospheric lines can reveal mass motions, and in particular the presence of a stellar wind and circumstellar material. Red giants in globular clusters were found to exhibit low velocity shifts in the cores of the H $\alpha$ or Na I D lines (cf. Peterson 1981; Bates et al. 1990, 1993); similarly, metal-poor field giants, which might be taken as the field counterparts of globular cluster giants, also indicate slow outflow from the asymmetries and line shifts in the $\mathrm{H} \alpha$, Ca II and Mg II lines (Smith et al. 1992; Dupree \& Smith 1995).

More recently, Lyons et al. (1996) discussed the Na I D and $\mathrm{H} \alpha$ stellar profiles for a sample of 63 RGB stars in 5 GC (M 4, M 13, M 22, M 55 and $\omega$ Cen), and found evidence of significant $\mathrm{Na}$ I D core shifts in $\sim 50 \%$ of the stars brighter than $\log L / L_{\odot} \sim 2.9$, whereas significant $\mathrm{H} \alpha$ core shifts were detected in $\sim 50 \%$ of the stars brighter than $\log L / L_{\odot} \sim 2.5$. These coreshifts are all $\leq 10 \mathrm{~km} \mathrm{~s}^{-1}$, i.e. much smaller than the escape velocity from the stellar photosphere $\left(\sim 50-60 \mathrm{~km} \mathrm{~s}^{-1}\right)$.

Two RGB stars in NGC 6752 were studied by Dupree et al. (1994), by a detailed analysis of the $\mathrm{Mg}$ II, Ca II K and $\mathrm{H} \alpha$ line profiles. These stars are at the RGB tip, and the $\mathrm{Ca}$ II $\mathrm{K}$ and $\mathrm{H} \alpha$ core shifts again revealed slow $\left(\leq 10 \mathrm{~km} \mathrm{~s}^{-1}\right)$ outflow motions. The asymmetries in the Mg II lines, however, indicated under certain assumptions a stellar wind with a terminal velocity of $\sim 150 \mathrm{~km} \mathrm{~s}^{-1}$, exceeding both the stellar photospheric escape velocity $\left(\sim 55 \mathrm{~km} \mathrm{~s}^{-1}\right)$ and the escape velocity from the cluster core $\left(\sim 23 \mathrm{~km} \mathrm{~s}^{-1}\right)$. The mass loss rate estimated by Dupree et al. (1994) from the $\mathrm{Mg}$ II results $\left(\sim 10^{-9} M_{\odot} \mathrm{yr}^{-1}\right)$ would lead to a total mass loss of $\sim 0.2 M_{\odot}$ over the star lifetime on the RGB $\left(\sim 2 \times 10^{8} \mathrm{yr}\right)$, in very good agreement with the expectations of the stellar evolution theory. This result alone is not sufficient to meet the requirements of the stellar evolution that all stars suffer some degree of mass loss during the phases preceding the HB. However, it shows that the mass loss phenomenon along the RGB does indeed occur, even if perhaps only occasionally and detected among the brightest stars, and it may be revealed using visual indicators, although less effectively and accurately than using chromospheric lines in the UV such as the Mg II, or in the near-IR such as the He I at $10830 \AA$ (Dupree et al. 1992).

The advent of the multi-fibre spectrograph FLAMES on VLT2-Kueyen (Pasquini et al. 2002), with a multiplex capability of 8 with UVES $(R=45000)$ and $\sim 130$ with GIRAFFE in MEDUSA mode $(R \sim 15000-30000)$, allows a much more efficient monitoring of visual diagnostics of mass outflow along

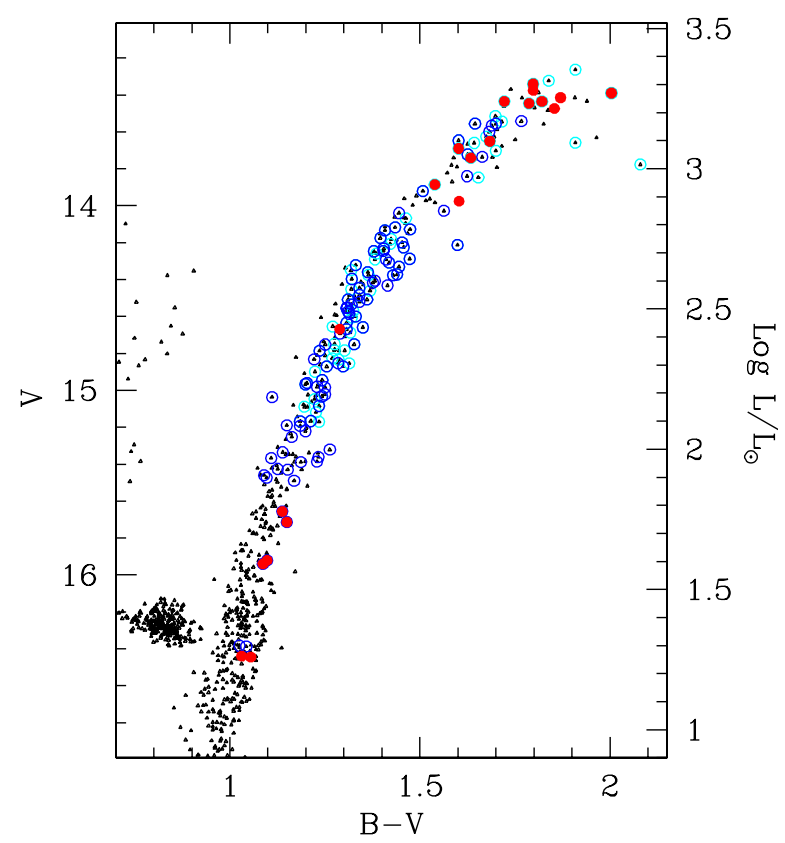

Fig. 1. Colour-magnitude diagram of NGC 2808 (Piotto et al. 2003) showing the 137 RGB stars observed with FLAMES. Filled circles indicate the stars observed with UVES, open circles indicate the stars observed with GIRAFFE/MEDUSA.

the RGB over a large magnitude range down from the RGB tip, especially in terms of sample size.

We have selected the globular cluster NGC 2808 for this monitoring, as it was the best candidate available for JanuaryFebruary observations, when Science Verification (SV) observations were scheduled.

\section{Observations and data reduction}

The target selection and observation were made possible by the accurate UBV photometry and astrometry provided by Piotto et al. (2003). All stars have been checked to be free from companions closer than 2.4 arcsec and brighter than $V+1.5$, where $V$ is the target magnitude. NGC 2808 is quite concentrated, and all target stars lie within a 7 ' radius from the cluster centre, and this has put to a difficult test the capabilities of the FLAMES fiber positioner (FLAMES has a corrected field of view of $25^{\prime}$ diameter).

We show in Fig. 1 the color-magnitude diagram of NGC 2808: the tip of the RGB is at $V=13.2$, corresponding to $M_{V} \sim-2.3, T_{\text {eff }} \sim 3800 \mathrm{~K}, \log L / L_{\odot} \sim 3.3$, and $M_{\text {bol }} \sim-3.5$. We have selected 13 RGB stars within the uppermost 0.8 mag interval and 7 more sampling 2.5 fainter magnitudes, to be observed with UVES ( $R=47000,8$ single fibres of 1 arcsec entrance aperture, grating centred at $580 \mathrm{~nm}$ and covering about $200 \mathrm{~nm}$ ). This wavelength range includes the $\mathrm{Na}$ I D and $\mathrm{H} \alpha$ lines. The exposure times were $1800 \mathrm{~s}$ for the stars with $V<14$ (except stars \#50119, 51499 and 56032 that were observed for $3600 \mathrm{~s}$ each), and $3 \times 3600 \mathrm{~s}$ for the stars with $V>14$ (except star \#53390 that was observed for $2 \times 3600$ s). Simultaneously, GIRAFFE in MEDUSA 
mode observed 117 more stars along the entire magnitude range with 3 setups, namely HR02 (Ca II H+K, $R=19600)$, HR12 (Na I D, $R=18700)$ and $\operatorname{HR} 14(\mathrm{H} \alpha, R=28800)$. The size of the MEDUSA fibres on the sky is 1.2 arcsec. The exposure times were $3 \times 3600 \mathrm{~s}$ with HR2, and $1800 \mathrm{~s}$ each with HR12 and HR14. Not all the stars were observed with all the MEDUSA setups due to the high crowding conditions and the difficulty of placing the fibers; about one fourth were observed only with the HR02 or HR12+HR14 setups, but more than 50 have complete coverage of the interesting spectral features, either entirely with the GIRAFFE setups, or in combination with the UVES spectra. Table 1 gives in the last column information on the setups used for each star. We note that this is the first time that such a large sample of globular cluster RGB stars have been observed in all the major, deep optical lines (i.e., $\mathrm{H} \alpha, \mathrm{Na} \mathrm{D}$ and $\mathrm{Ca}$ II $\mathrm{H}$ and $\mathrm{K}$ ) that are normally used to study the presence of chromospheres and/or mass motions in the atmospheres.

Given the large interval in magnitude, $S / N$ ratios vary a lot. In the case of the UVES spectra, $S / N$ measured near $630 \mathrm{~nm}$ is about 100 for the brighter stars, and about 40 for the fainter ones, with 120 and 20 being the extremes. In the $\mathrm{Ca}$ II region $S / N$ 's were quite low (i.e. about 15 at the bottom of the K line for the brightest objects), while in the $\mathrm{Na}$ I D and $\mathrm{H} \alpha$ setups they varied from about 25 to 150 , following the luminosity distribution, with peaks around 80-100, and 50-60, respectively.

We collect the photometric information for our target stars in Table 1, including the IR photometry that is now available for all of our targets - except 6 - in the 2MASS AllSky Data Release (accessible at www.ipac.caltech.edu/ 2mass/releases/allsky/ and released on March 25, 2003).

The observations were performed during SV between January 24 and February 2, 2003. Most of the data were reduced by ESO personnel using the standard pipelines for FLAMES (see Pasquini et al. 2002 for a description), and were made public, according to ESO SV policies, on March 3, 2003. These reductions were intended to give a first insight on the data, and were not deemed optimal by the SV team: e.g., offsets in wavelegth calibrations between fibers could be present, in some cases producing errors of up to $\pm 10 \mathrm{~km} \mathrm{~s}^{-1}$ in radial velocities ( $R V$ 's). We inspected our spectra and found them quite suitable for our purposes in two of the three GIRAFFE setups (HR02 and 12). The wavelength calibration for the $\mathrm{H} \alpha$ setup HR14 was not quite so satisfactory, but no re-reduction of the HR14 spectra was performed since the GIRAFFE pipeline was still affected by a number of problems. However, for our purpose we mostly need to analyse $\mathrm{H} \alpha$ profiles, and an accurate wavelength calibration over the entire wavelength range of HR14 is not essential, as long as it can be trusted over a small wavelength range encompassing $\mathrm{H} \alpha$ itself and a few close photospheric lines. We then defined a pseudo- $R V$ based on a few photospheric lines in the immediate vicinity of $\mathrm{H} \alpha$, shifted to zero $R V$ all the spectra, and retained for further analysis only the $50 \AA$ centred on $\mathrm{H} \alpha$. No attempt to derive centre/core shifts (see Sect. 4) was done on these spectra, but they are perfectly suitable to search for emissions. Because of these problems with wavelength calibration the subtraction of the sky contribution from the GIRAFFE spectra was not advisable. Direct contamination from sky lines could be excluded since the radial velocity of the cluster is $\sim 100 \mathrm{~km} \mathrm{~s}^{-1}$. However, scattered sky light can in principle affect the line profiles, so we have estimated carefully the counts from the sky (using the dedicated fibres) and from the continuum and line core at $\mathrm{H} \alpha$ for a few stars in the critical magnitude range $V=14.0$ to 15.0 . For brighter stars the sky contamination becomes irrelevant, and for fainter stars no $\mathrm{H} \alpha$ emission was detected (see Sect. 4.1.1). For homogeneity, we have treated the UVES spectra in the same way. We have thus estimated that neglecting the contribution of the sky scattered light can introduce an error of $\sim 2 \%$ with UVES at $V=14$, and of $\sim 1-2 \%$ with GIRAFFE at $V=$ 14.5-15.0. These are our thresholds for detecting $\mathrm{H} \alpha$ emission, which appears to be much stronger than these values (see Sect. 4.1.1), therefore we are confident that neglecting the sky contribution in our analysis has not introduced any significant bias in our results.

As for the UVES spectra, we decided to reduce them again using the available UVES pipeline in order to obtain the best possible accuracy. FLAMES/UVES data require a rather careful treatment in order to remove both instrumental effects common to any high resolution, cross-dispersed echelle spectrograph and some specific ones due to its use in multi-object, fibre-fed mode. An ad hoc Data Reduction Software (DRS) was specifically developed for this purpose, in the form of a set of procedures which are available as a context of the Münich Image Data Analysis System (MIDAS).

This DRS, described in detail elsewhere (Mulas et al. 2002), makes use of various flat-field exposures, taken both in fibre and in slit mode, to separately derive the pixel to pixel response of the detector and the contribution of each fibre to the overall distribution of light on the resulting two-dimensional science frame. The precise position of the orders and fibres is measured on the science frame itself. These pieces of information are then used together to perform an optimal extraction which, at the same time, carefully corrects for light contamination between fibres whose dispersed images are adjacent on the science frame.

Optimal extraction maximises the signal to noise ratio, since it does not discard the "wings" of the cross-dispersion point spread function (PSF) of the fibres, and effectively detects and removes most cosmic ray hits.

Exposures of a reference Th-Ar calibration lamp were extracted in exactly the same way as the science frames, to ensure the maximum coherence, and a 2-dimensional polynomial fit was performed on the detected lines to obtain the dispersion function of the spectrograph, separately for each fibre. The reference $\mathrm{Th}-\mathrm{Ar}$ exposures were taken on the same day as the science data to be calibrated with them. The resulting wavelength calibration error resulted to be well below $0.01 \AA$ throughout the wavelength range. Science spectra were rebinned to wavelength space and the echelle orders merged, making a weighted average where they overlap. Pixel by pixel variances were propagated by the DRS throughout the reduction procedure. 
Table 1. List of RGB stars observed with FLAMES during Science Verification on Jan. 24-Feb. 02, 2003. Identification, coordinates and visual photometry are taken from Piotto et al. (2003), IR photometry is taken from the 2MASS Catalog.

\begin{tabular}{|c|c|c|c|c|c|c|c|c|}
\hline ID No. & $\mathrm{RA}(2000)$ & $\operatorname{Dec}(2000)$ & $B$ & $V$ & $J$ & $H$ & $K$ & Notes \\
\hline 7183 & 91202.8710 & -644934.069 & 16.168 & 14.854 & & & & 2 \\
\hline 7536 & 91231.7065 & -644922.268 & 15.812 & 14.372 & 11.802 & 11.069 & 10.829 & 1,2 \\
\hline 7558 & 91220.1287 & -644921.891 & 16.576 & 15.389 & 13.160 & 12.518 & 12.407 & 1 \\
\hline 7788 & 91157.1979 & -644914.551 & 16.168 & 14.870 & 12.472 & 11.777 & 11.623 & 1,2 \\
\hline 8603 & 91214.0510 & -644842.915 & 15.847 & 14.432 & 11.902 & 11.162 & 10.957 & 1 \\
\hline 8679 & 91144.5585 & -644839.890 & 16.164 & 14.961 & 12.753 & 12.156 & 12.022 & $1,2, F$ \\
\hline 8739 & 91151.2018 & -644837.535 & 15.761 & 14.288 & 11.620 & 10.813 & 10.693 & 1,2 \\
\hline 8826 & 91209.8073 & -644833.243 & 16.275 & 15.033 & 12.746 & 12.057 & 11.911 & 1,2 \\
\hline 9230 & 91213.3528 & -644811.617 & 15.591 & 14.028 & 11.252 & 10.449 & 10.303 & 1 \\
\hline 9724 & 91200.9163 & 644743.204 & 15.869 & 14.508 & 11.994 & 11.282 & 11.090 & 1,2 \\
\hline 9992 & 91246.8254 & -644723.732 & 15.402 & 13.702 & 10.729 & 9.886 & 9.659 & 2 \\
\hline 10012 & 91158.5661 & -644723.138 & 15.682 & 14.225 & 11.613 & 10.836 & 10.679 & 1,2 \\
\hline 10105 & 91221.1477 & -644713.906 & 15.976 & 14.669 & 12.279 & 11.564 & 11.417 & 1,2 \\
\hline 10201 & 91245.7573 & -644705.388 & 16.864 & 15.714 & 13.508 & 12.868 & 12.759 & 1,3 \\
\hline 10265 & 91156.6812 & -644700.535 & 15.728 & 14.309 & 11.772 & 11.026 & 10.823 & 1,2 \\
\hline 10341 & 91225.4161 & -644652.409 & 16.009 & 14.659 & 12.205 & 11.526 & 11.335 & 1,2 \\
\hline 10571 & 91241.1223 & -644625.848 & 15.806 & 14.376 & 11.771 & 10.988 & 10.844 & 1,2 \\
\hline 10681 & 91205.7235 & -644613.801 & 15.261 & 13.545 & 10.635 & 9.855 & 9.586 & 2 \\
\hline 13575 & 91131.6300 & -644902.281 & 16.615 & 15.386 & 13.090 & 12.417 & 12.270 & 1 \\
\hline 13983 & 91139.7915 & -644825.463 & 17.027 & 15.940 & 13.832 & 13.246 & 13.092 & 1,3 \\
\hline 30523 & 91122.4875 & -645523.351 & 16.658 & 15.489 & 13.320 & 12.697 & 12.550 & 1 \\
\hline 30763 & 91131.6195 & -645457.989 & 15.927 & 14.606 & 12.287 & 11.577 & 11.465 & 2 \\
\hline 30900 & 91136.3238 & -645447.078 & 16.319 & 15.084 & 12.890 & 12.203 & 12.075 & 1,2 \\
\hline 30927 & 91141.3502 & -645445.153 & 15.215 & 13.516 & 10.814 & 9.985 & 9.802 & 2 \\
\hline 31851 & 91114.3696 & -645336.046 & 16.420 & 15.221 & 13.064 & 12.411 & 12.280 & 1 \\
\hline 32398 & 91139.4612 & -645301.270 & 16.004 & 14.687 & 12.321 & 11.590 & 11.483 & 2 \\
\hline 32469 & 91116.3279 & -645255.955 & 15.485 & 14.040 & 11.531 & 10.777 & 10.689 & 1 \\
\hline 32685 & 91127.0742 & -645244.375 & 16.794 & 15.656 & 13.548 & 12.956 & 12.772 & 1,3 \\
\hline 32862 & 91142.0399 & -645234.140 & 16.379 & 15.166 & 12.871 & 12.171 & 11.999 & 1,2 \\
\hline 32909 & 91139.6760 & -645231.924 & 16.272 & 15.022 & 12.710 & 12.046 & 11.910 & 1 \\
\hline 32924 & 91122.2965 & -645231.081 & 16.138 & 14.853 & 12.515 & 11.785 & 11.701 & 1 \\
\hline 33452 & 91140.7982 & -645201.561 & 16.405 & 15.170 & 12.970 & 12.271 & 12.167 & 2 \\
\hline 33918 & 91101.6928 & -645136.087 & 15.776 & 14.331 & 11.756 & 11.021 & 10.887 & 1,2 \\
\hline 34008 & 91127.5242 & -645131.290 & 16.346 & 15.119 & 12.894 & 12.240 & 12.115 & 2 \\
\hline 34013 & 91123.8497 & -645130.965 & 17.499 & 16.444 & 14.503 & 13.897 & 13.843 & 3 \\
\hline 35265 & 91122.7197 & -645012.117 & 16.584 & 15.321 & 12.965 & 12.287 & 12.180 & 1 \\
\hline 37496 & 91213.8815 & -645709.411 & 16.126 & 14.871 & 12.586 & 11.891 & 11.730 & 1,2 \\
\hline 37505 & 91226.4217 & -645708.391 & 16.058 & 14.783 & 12.471 & 11.739 & 11.591 & 2 \\
\hline 37781 & 91213.0342 & -645641.142 & 16.148 & 15.037 & 13.001 & 12.396 & 12.307 & $1, \mathrm{~F}$ \\
\hline 37872 & 91223.0712 & -645634.303 & 15.334 & 13.650 & 10.767 & 9.904 & 9.711 & 2,3 \\
\hline 37998 & 91212.5554 & -645624.603 & 15.638 & 14.233 & 11.706 & 10.960 & 10.796 & 1,2 \\
\hline 38228 & 91230.9696 & -645608.521 & 16.211 & 14.981 & 12.700 & 12.035 & 11.887 & 1 \\
\hline 38244 & 91208.9333 & -645607.955 & 16.378 & 15.193 & 12.996 & 12.307 & 12.177 & 1 \\
\hline 38559 & 91256.1453 & -645548.356 & 15.541 & 14.133 & 11.674 & 10.915 & 10.754 & $1,2, F$ \\
\hline 38660 & 91239.8673 & -645543.083 & 15.772 & 14.452 & 11.995 & 11.407 & 9.519 & 2 \\
\hline 38967 & 91208.8682 & -645527.764 & 16.415 & 15.252 & 13.060 & 12.383 & 12.297 & 1 \\
\hline 39060 & 91214.5222 & -645524.085 & 15.793 & 14.417 & 11.939 & 11.193 & 11.036 & 1 \\
\hline 39577 & 91210.9820 & -645503.959 & 15.820 & 14.509 & 12.085 & 11.357 & 11.189 & $1,2, \mathrm{~F}$ \\
\hline 40196 & 91205.9806 & -645443.265 & 15.894 & 14.570 & 12.142 & 11.440 & 11.264 & 2 \\
\hline 40983 & 91148.6726 & -645420.737 & 15.723 & 14.360 & 11.906 & 11.153 & 10.988 & 1,2 \\
\hline 41008 & 91217.6744 & -645419.865 & 16.339 & 15.189 & 12.973 & 12.309 & 12.200 & 1 \\
\hline 41828 & 91208.5555 & -645400.670 & 15.862 & 14.521 & 12.129 & 11.316 & 11.210 & 1 \\
\hline 41969 & 91150.8999 & -645357.615 & 15.787 & 14.446 & 11.972 & 11.254 & 11.092 & 1,2 \\
\hline 42073 & 91216.2176 & -645355.186 & 15.702 & 14.291 & 11.733 & 11.031 & 10.783 & 1 \\
\hline
\end{tabular}


Table 1. continued.

\begin{tabular}{|c|c|c|c|c|c|c|c|c|}
\hline ID No. & $\overline{\mathrm{RA}(2000)}$ & $\overline{\operatorname{Dec}(2000)}$ & $\overline{\bar{B}}$ & $\overline{\bar{V}}$ & $\overline{\bar{J}}$ & $\overline{\bar{H}}$ & $\overline{\bar{K}}$ & $\overline{\text { Notes }}$ \\
\hline 42165 & 91233.7942 & -645352.922 & 17.430 & 16.387 & 14.369 & 13.816 & 13.613 & 1 \\
\hline 42789 & 91148.9406 & -645341.364 & 16.476 & 15.367 & 13.246 & 12.610 & 12.507 & 1 \\
\hline 42886 & 91259.1095 & -645338.608 & 17.019 & 15.921 & 13.780 & 13.157 & 13.040 & 1,3 \\
\hline 42996 & 91225.0560 & -645337.226 & 16.552 & 15.426 & 13.294 & 12.656 & 12.523 & 1 \\
\hline 43041 & 91240.0467 & -645335.930 & 15.349 & 13.723 & 10.869 & 10.063 & 9.822 & 1,2 \\
\hline 43217 & 91232.6714 & -645332.870 & 17.471 & 16.440 & 14.454 & 13.805 & 13.675 & 3 \\
\hline 43247 & 91210.1184 & -645332.654 & 16.022 & 14.786 & 12.486 & 11.732 & 11.543 & 1,2 \\
\hline 43281 & 91221.2449 & -645332.058 & 16.270 & 15.047 & & & & 2 \\
\hline 43333 & 91255.0733 & -645330.392 & 16.570 & 15.473 & 13.408 & 12.776 & 12.698 & $1, \mathrm{~F}$ \\
\hline 43561 & 91214.4769 & -645326.725 & 15.162 & 13.323 & 10.177 & 9.295 & 9.073 & 2 \\
\hline 43794 & 91226.3414 & -645322.607 & 15.861 & 14.554 & 12.157 & 11.468 & 11.271 & 1,2 \\
\hline 43822 & 91158.4947 & -645322.341 & 15.931 & 14.600 & 12.176 & 11.422 & 11.291 & 1,2 \\
\hline 44573 & 91206.1145 & -645308.983 & 16.285 & 15.089 & 12.709 & 12.022 & 11.912 & 2 \\
\hline 44665 & 91215.2786 & -645307.367 & 15.649 & 14.245 & 11.718 & 10.980 & 10.799 & 1,2 \\
\hline 44716 & 91217.8493 & -645306.449 & 16.123 & 14.899 & 12.605 & 11.924 & 11.748 & 2 \\
\hline 44984 & 91245.8780 & -645301.411 & 15.855 & 14.535 & 12.088 & 11.340 & 11.219 & 1,2 \\
\hline 45162 & 91159.2356 & -645259.262 & 15.172 & 13.263 & 10.019 & 9.074 & 8.853 & 2 \\
\hline 45443 & 91221.3723 & -645254.137 & 15.885 & 14.574 & 12.128 & 11.385 & 11.283 & 1,2 \\
\hline 45840 & 91211.1152 & -645247.477 & 15.552 & 14.117 & 11.516 & 10.703 & 10.536 & 1,2 \\
\hline 46041 & 91202.9671 & -645244.370 & 16.166 & 14.967 & 12.683 & 12.008 & 11.850 & 1,2 \\
\hline 46099 & 91233.5788 & -645243.088 & 15.375 & 13.741 & 10.915 & 10.044 & 9.835 & 2,3 \\
\hline 46367 & 91200.4581 & -645238.810 & 16.054 & 14.832 & 12.526 & 11.884 & 11.485 & 1 \\
\hline 46422 & 91156.0932 & -645237.896 & 15.174 & 13.376 & & & & 3 \\
\hline 46580 & 91156.1904 & -645235.387 & 15.292 & 13.690 & & & & 2,3 \\
\hline 46663 & 91217.0998 & -645233.946 & 15.860 & 14.551 & 12.146 & 11.456 & 11.323 & 1 \\
\hline 46726 & 91150.5738 & -645233.000 & 15.740 & 14.375 & 11.910 & 11.160 & 10.998 & 2 \\
\hline 46868 & 91209.7828 & -645230.452 & 16.003 & 14.752 & & & & 1 \\
\hline 46924 & 91204.6008 & -645229.587 & 16.096 & 14.826 & & & & 2 \\
\hline 47031 & 91200.9644 & -645227.851 & 15.605 & 14.181 & 11.593 & 10.894 & 10.355 & 2 \\
\hline 47145 & 91158.1949 & -645225.936 & 15.249 & 13.647 & 10.814 & 9.995 & 9.777 & 1,2 \\
\hline 47421 & 91156.4377 & -645221.270 & 15.571 & 14.175 & 11.674 & 10.834 & 10.696 & 1,2 \\
\hline 47452 & 91220.2257 & -645220.564 & 15.857 & 13.777 & 10.268 & 9.499 & 9.157 & 2 \\
\hline 47606 & 91206.6571 & -645218.225 & 15.257 & 13.436 & 10.227 & 9.349 & 9.103 & 2,3 \\
\hline 48011 & 91148.0108 & -645212.236 & 15.718 & 14.397 & 11.965 & 11.233 & 11.040 & 1,2 \\
\hline 48060 & 91155.1995 & -645211.458 & 15.256 & 13.556 & 10.609 & 9.741 & 9.523 & 1,2 \\
\hline 48128 & 91152.1753 & -645210.603 & 15.825 & 14.484 & 11.997 & 11.292 & 11.118 & 1,2 \\
\hline 48424 & 91206.6195 & -645205.871 & 15.772 & 14.452 & 11.995 & 11.407 & 9.519 & 2 \\
\hline 48609 & 91216.6443 & -645202.923 & 15.285 & 13.415 & 10.229 & 9.353 & 9.101 & 3 \\
\hline 48889 & 91208.5086 & -645158.467 & 15.139 & 13.341 & 10.340 & 9.431 & 9.253 & 2,3 \\
\hline 49509 & 91156.2108 & -645149.116 & 15.429 & 13.921 & 11.237 & 10.379 & 10.250 & 1 \\
\hline 49680 & 91210.0583 & -645146.474 & 15.256 & 13.567 & 10.573 & 9.706 & 9.497 & 1 \\
\hline 49743 & 91236.7974 & -645145.097 & 16.353 & 15.168 & 12.925 & 12.266 & 12.159 & 1,2 \\
\hline 49753 & 91206.8961 & -645145.474 & 15.669 & 14.350 & 11.804 & 11.018 & 10.886 & 2 \\
\hline 49942 & 91212.0864 & -645142.617 & 15.400 & 13.736 & 10.848 & 10.027 & 9.812 & 1 \\
\hline 50119 & 91142.9184 & -645139.752 & 15.425 & 13.886 & 11.210 & 10.382 & 10.218 & 2,3 \\
\hline 50371 & 91207.9720 & -645135.858 & 15.464 & 13.840 & 10.999 & 10.144 & 9.976 & 1 \\
\hline 50561 & 91223.1839 & -645132.695 & 16.120 & 14.834 & 12.428 & 11.725 & 11.548 & 2 \\
\hline 50681 & 91218.4772 & -645130.760 & 15.303 & 13.660 & 10.874 & 10.100 & 9.905 & 2 \\
\hline 50761 & 91157.0729 & -645129.676 & 15.394 & 13.390 & 9.947 & 9.096 & 8.827 & 2,3 \\
\hline 50861 & 91211.0865 & -645127.889 & 15.201 & 13.556 & 10.727 & 9.851 & 9.662 & 1,2 \\
\hline 50866 & 91154.2702 & -645127.928 & 15.941 & 14.634 & 12.219 & 11.524 & 11.353 & 1 \\
\hline 50910 & 91142.6346 & -645127.221 & 16.234 & 14.984 & 12.697 & 12.033 & 11.877 & 1 \\
\hline 51416 & 91229.2944 & -645118.935 & 16.188 & 14.945 & 12.619 & 11.911 & 11.803 & 1,2 \\
\hline 51454 & 91202.2715 & -645118.513 & 15.233 & 13.446 & 10.336 & 9.492 & 9.244 & 2,3 \\
\hline 51499 & 91207.3496 & -645117.800 & 15.157 & 13.435 & 10.447 & 9.567 & 9.382 & 2,3 \\
\hline 51515 & 91159.3832 & -645117.525 & 15.812 & 14.213 & 11.472 & 10.665 & 10.525 & 1,2 \\
\hline 51646 & 91150.3432 & -645115.395 & 16.550 & 15.460 & 13.425 & 12.791 & 12.668 & 1 \\
\hline 51871 & 91205.4500 & -645111.901 & 15.309 & 13.542 & 10.040 & 9.718 & 9.514 & 1 \\
\hline
\end{tabular}


Table 1. continued.

\begin{tabular}{|c|c|c|c|c|c|c|c|c|}
\hline ID No. & RA(2000) & $\operatorname{Dec}(2000)$ & $B$ & $V$ & $J$ & $H$ & $K$ & Notes \\
\hline 51930 & 91149.9340 & -645110.872 & 15.531 & 14.068 & 11.422 & 10.655 & 10.478 & 2 \\
\hline 51963 & 91227.6355 & -645110.138 & 15.652 & 14.199 & 11.577 & 10.821 & 10.652 & 1,2 \\
\hline 51983 & 91202.5038 & -645110.068 & 15.328 & 13.474 & 10.294 & 9.391 & 9.182 & 3 \\
\hline 52006 & 91218.1017 & -645109.524 & 15.787 & 14.446 & 11.972 & 11.254 & 11.092 & 1,2 \\
\hline 52048 & 91205.7704 & -645180.996 & 15.645 & 14.259 & 10.387 & 11.012 & 10.797 & 2 \\
\hline 52647 & 91212.2817 & -645059.590 & 15.925 & 14.654 & 12.293 & 11.592 & 11.432 & 2 \\
\hline 53284 & 91201.4868 & -645049.350 & 15.501 & 13.847 & 10.902 & 9.254 & 9.953 & 2 \\
\hline 53390 & 91152.9455 & -645047.860 & 15.958 & 14.669 & 12.271 & 11.573 & 11.379 & 3 \\
\hline 53579 & 91148.7436 & -645044.801 & 15.830 & 14.461 & 11.877 & 11.178 & 10.992 & 2 \\
\hline 54264 & 91221.5127 & -645033.737 & 15.285 & 13.415 & 10.229 & 9.353 & 9.101 & 1 \\
\hline 54284 & 91154.7689 & -645033.662 & 15.789 & 14.407 & 11.877 & 11.148 & 10.985 & 1 \\
\hline 54308 & 91201.6780 & -645033.286 & 15.568 & 13.659 & 10.182 & 9.264 & 9.024 & 2 \\
\hline 54733 & 91207.8037 & -645026.397 & 15.602 & 14.128 & 11.523 & 10.761 & 10.571 & 1,2 \\
\hline 54756 & 91146.3335 & -645026.044 & 16.086 & 14.784 & 12.416 & 11.679 & 11.528 & 2 \\
\hline 54789 & 91200.4239 & -645025.567 & 15.626 & 14.247 & 11.756 & 11.019 & 10.863 & 1,2 \\
\hline 55031 & 91158.1972 & -645021.703 & 15.283 & 13.600 & 10.680 & 9.813 & 9.620 & 1 \\
\hline 55354 & 91210.3337 & -645016.108 & 16.024 & 14.749 & 12.406 & 11.655 & 11.531 & 2 \\
\hline 55437 & 91211.4613 & -645014.694 & 15.982 & 14.692 & 12.302 & 11.586 & 11.420 & 1 \\
\hline 55609 & 91229.9315 & -645011.384 & 16.078 & 14.751 & 12.301 & 11.575 & 11.467 & 1,2 \\
\hline 55627 & 91205.4152 & -645011.404 & 15.899 & 14.585 & 12.188 & 11.524 & 11.338 & 1 \\
\hline 56032 & 91145.5811 & -645004.209 & 15.579 & 13.976 & 11.112 & 10.292 & 10.095 & 3 \\
\hline 56136 & 91230.9806 & -645002.010 & 17.408 & 16.383 & 14.434 & 13.879 & 13.608 & 1 \\
\hline 56536 & 91204.3411 & -644955.639 & 15.301 & 13.626 & 10.785 & 9.896 & 9.688 & 2 \\
\hline 56710 & 91230.1594 & -644952.049 & 16.582 & 15.430 & 13.232 & 12.553 & 12.396 & 1 \\
\hline 56924 & 91215.1423 & -644948.859 & 15.629 & 14.207 & 11.587 & 10.869 & 10.676 & 2 \\
\hline
\end{tabular}

Notes: 1 = MEDUSA HR12 + HR14; 2 = MEDUSA HR02; 3 = UVES; F = field star.

Both UVES and GIRAFFE spectra were then analysed using IRAF $^{1}$ and ISA (Gratton 1988) to measure equivalent widths ( $E W$ 's) and $R V$ 's. The $R V$ 's were measured from the Doppler shifts of selected photospheric lines, mostly of Fe I, separately in the GIRAFFE setups (using rvidlines in IRAF on about 20 to 30 lines), and in the UVES spectra (using the ISA package). Errors are $\sim 0.6 \mathrm{~km} \mathrm{~s}^{-1}$ for UVES and $\sim 1.5 \mathrm{~km} \mathrm{~s}^{-1}$ for GIRAFFE. The observed $R V$ 's were used to shift all spectra to zero radial velocity, thus eliminating any residual possible problem with the zero point shifts due to the non optimal calibration, which we found almost non existing anyway for the HR02 and 12 setups. Multiple exposures of the same star were coadded to enhance the $S / N$.

In a few cases the derived $R V$ was strongly discrepant from the bulk of the other stars; this is a real effect, as confirmed by the spectral ranges where atmospheric or interstellar lines are present (e.g. the Na I D lines). We have assumed that those are field objects and have marked them with " $F$ " in Table 1. They have not been included in the following analysis to avoid introducing spurious effects.

\footnotetext{
${ }^{1}$ IRAF is distributed by the NOAO, which are operated by AURA, under contract with NSF.
}

From the 20 stars observed with UVES we derive a mean heliocentric $R V$ of $100.9 \mathrm{~km} \mathrm{~s}^{-1}(\sigma=8.5)$, whereas from the lower precision GIRAFFE measures we obtain $102.1 \mathrm{~km} \mathrm{~s}^{-1}$ $(\sigma=10.9)$ using 81 member stars observed in the $\mathrm{Na}$ I region, and $99.0 \mathrm{~km} \mathrm{~s}^{-1}(\sigma=10.3)$ using 83 stars observed in the Ca II setup.

The first spectroscopic observations of NGC 2808 were taken by Kinman nearly 50 years ago: he set on a bright star in the center and exposed for about $3 \mathrm{~h}$, but because of guiding uncertainties the spectrum obtained was at least partially an integrated spectrum (Kinman 2003, private communication). The mean radial velocity derived from these data was $101 \pm$ $5 \mathrm{~km} \mathrm{~s}^{-1}$ (Kinman 1959). Our results are in excellent agreement with this earliest determination. Later measures of mean radial velocity, summarised by Harris (1996, updated 2003) as $93.6 \pm 2.4 \mathrm{~km} \mathrm{~s}^{-1}$, include values ranging from e.g. $80.1 \pm$ 9.9 (Rutledge et al. 1997), to $98 \pm 4$ (Hesser et al. 1986), to $104.1 \pm 4.4$ (Webbink 1981) $\mathrm{km} \mathrm{s}^{-1}$. The most discrepant result, by Rutledge et al. (1997), should probably be given little weight, as $\sim 25 \%$ of their results on globular clusters differ by more than $20 \mathrm{~km} \mathrm{~s}^{-1}$ from the average of all previous determinations, and the authors say that the goal of their project was not to determine accurate cluster velocities. 

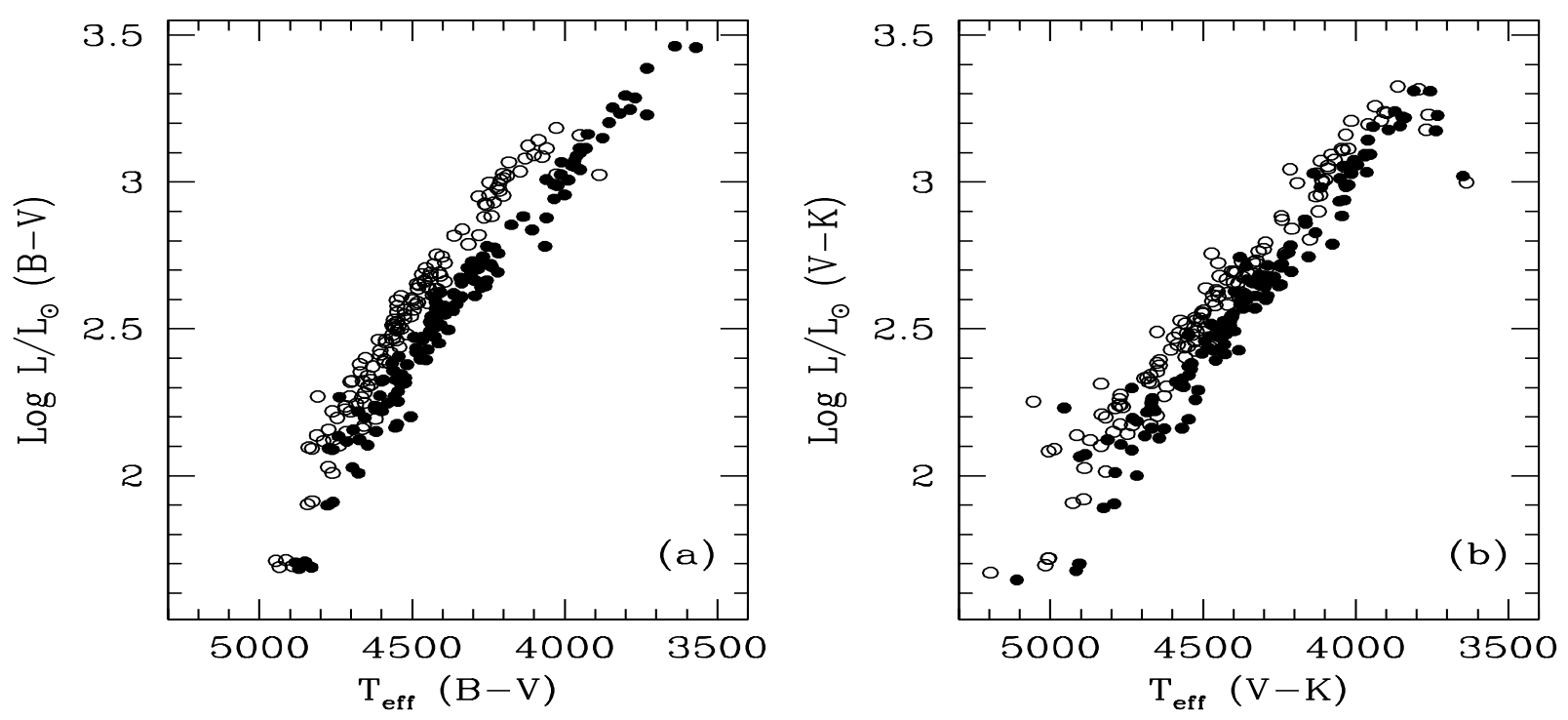

Fig. 2. Comparison of the temperatures and luminosities obtained using the Montegriffo et al. (1998, open circles) and Alonso et al. (1999, 2001, filled dots) calibrations, for the $(B-V)$ colours (panel a)) and $(V-K)$ colours (panel b)).

Therefore all determinations, excluding Rutledge et al. (1997), are in agreement within 1- $\sigma$ error.

\section{The physical and atmospheric parameters}

Assuming for NGC 2808 the reddening $E(B-V)=0.22$ and apparent distance modulus $(m-M)_{V}=15.59$ (Harris 1996), we have calculated the intrinsic colours $(B-V)_{0}$ and $(V-K)_{0}$ and absolute magnitudes of our stars using the relations: $E(V-K)=2.75 E(B-V), \mathrm{A}_{V}=3.1 E(B-V)$ and $\mathrm{A}_{K}=$ $0.35 E(B-V)$ (Cardelli et al. 1989).

The effective temperatures and bolometric corrections have been obtained both from visual and IR colours using two independent empirical calibrations specifically derived for late type giant stars by Montegriffo et al. (1998, their Table 3) and Alonso et al. (1999, their Eqs. (4), (9) and (17). See also Alonso et al. 2001). The metallicity assumed for NGC 2808 is $[\mathrm{Fe} / \mathrm{H}]=-1.25$ as an intermediate value among several determinations (cf. Walker 1999).

Transformations of the 2MASS $\mathrm{K}$ data to the ESO and TCS photometric systems, used by Montegriffo and Alonso respectively, have been performed using the relations provided by Carpenter (2001). The relations we have used are: $K_{\mathrm{ESO}}=$ $K_{2 \mathrm{MASS}}-0.005(J-K)_{2 \mathrm{MASS}}+0.045$ and $K_{\mathrm{TCS}}=K_{2 \mathrm{MASS}}+$ $0.006(J-K)_{2 \mathrm{MASS}}+0.002$. The relation for the TCS photometric system has been obtained via an intermediate passage through the CIT system.

The $T_{\text {eff }}$ 's obtained from these two calibrations are compared in Fig. 2, where we see that they are quite compatible once allowance is made for a systematic offset that makes Alonso temperatures lower by $\simeq 153(\sigma=65)$ and $88(\sigma=$ $17) \mathrm{K}$, when considering $T_{\text {eff }}$ 's derived from $(B-V)$ and $(V-K)$ respectively.

This difference is irrelevant for the purpose of the present paper, where temperatures and luminosities are only used to locate correctly the stars in the HR diagram. It might be more relevant for a careful estimate of elemental abundances, but this

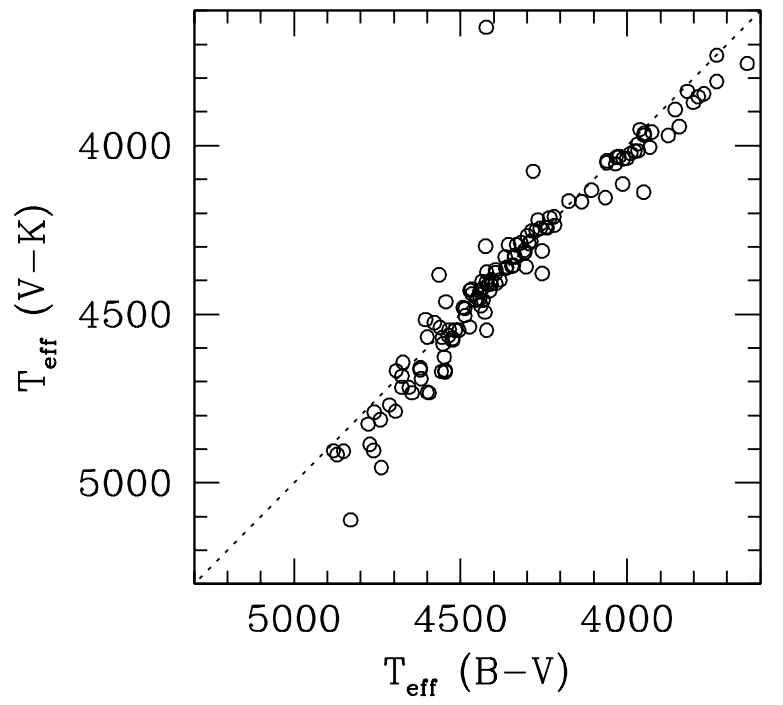

Fig. 3. Comparison of the temperatures obtained from the $(B-V)$ and $(V-K)$ colours, using Alonso et al. $(1999,2001)$ calibration.

aspect is discussed elsewhere (Carretta et al. 2003). In the following we shall use Alonso et al. $(1999,2001)$ calibration as it is widely used and is therefore more convenient for comparison with other studies. With this calibration, the $(B-V)$ and $(V-K)$ colours yield similar results, except at the ends of the temperature range we are interested in, as we can see in Fig. 3; the average difference between the two $T_{\text {eff }}$ 's is $19(\sigma=$ 95) $\mathrm{K}$.

We list in Table 2 the values of temperature and luminosity we obtain for all our stars from the $(B-V)$ and $(V-K)$ colours and the Alonso et al. $(1999,2001)$ calibration, assuming $M_{\mathrm{bol}, \odot}=4.75$. The values of gravity from the two colours are the same within $0.05 \mathrm{dex}$ in the logarithm, and we list only the values derived from the $(B-V)$ colours. 
Table 2. Physical and atmospheric parameters for our RGB stars in NGC 2808. The last three columns give the measured heliocentric radial velocities for two GIRAFFE setups (HR02 and 12) and for UVES, derived by averaging several photospheric lines.

\begin{tabular}{|c|c|c|c|c|c|c|c|c|c|c|}
\hline ID No. & $M_{V}$ & $\overline{(B-V)_{0}}$ & $\begin{array}{c}T_{\text {eff }} \\
(B-V)\end{array}$ & $\begin{array}{c}\log L \\
(B-V)\end{array}$ & $\begin{array}{c}\log g \\
(B-V)\end{array}$ & $\begin{array}{c}T_{\text {eff }} \\
(V-K)\end{array}$ & $\begin{array}{c}\log L \\
(V-K)\end{array}$ & $\begin{array}{r}R V_{02} \\
\mathrm{~km} \mathrm{~s}^{-1}\end{array}$ & $\begin{array}{r}R V_{12} \\
\mathrm{~km} \mathrm{~s}^{-1}\end{array}$ & $\begin{array}{r}R V_{\mathrm{U}} \\
\mathrm{km} \mathrm{s}^{-1}\end{array}$ \\
\hline 7183 & -0.736 & 1.094 & 4432 & 2.405 & 1.50 & & & 100.31 & & \\
\hline 7536 & -1.218 & 1.220 & 4261 & 2.645 & 1.19 & 4245 & 2.650 & 93.65 & 98.83 & \\
\hline 7558 & -0.201 & 0.967 & 4618 & 2.150 & 1.83 & 4691 & 2.135 & & 121.32 & \\
\hline 7788 & -0.720 & 1.078 & 4454 & 2.394 & 1.52 & 4459 & 2.392 & 89.00 & 96.35 & \\
\hline 8603 & -1.158 & 1.195 & 4294 & 2.612 & 1.24 & 4290 & 2.612 & & 110.88 & \\
\hline 8739 & -1.302 & 1.253 & 4219 & 2.692 & 1.13 & 4211 & 2.695 & 110.92 & 111.89 & \\
\hline 8826 & -0.557 & 1.022 & 4536 & 2.310 & 1.64 & 4564 & 2.303 & 95.47 & 98.45 & \\
\hline 9230 & -1.562 & 1.343 & 4107 & 2.836 & 0.94 & 4132 & 2.827 & & 89.31 & \\
\hline 9724 & -1.082 & 1.141 & 4366 & 2.561 & 1.32 & 4330 & 2.570 & 84.83 & 84.33 & \\
\hline 9992 & -1.888 & 1.480 & 3949 & 3.041 & 0.67 & 3964 & 3.033 & 105.77 & & \\
\hline 10012 & -1.365 & 1.237 & 4239 & 2.711 & 1.12 & 4243 & 2.710 & 103.83 & 104.26 & \\
\hline 10105 & -0.921 & 1.087 & 4442 & 2.477 & 1.44 & 4455 & 2.474 & 81.11 & 81.58 & \\
\hline 10201 & 0.124 & 0.930 & 4676 & 2.008 & 1.99 & 4717 & 2.000 & & 111.21 & 105.96 \\
\hline 10265 & -1.281 & 1.199 & 4289 & 2.662 & 1.19 & 4283 & 2.664 & 106.50 & 108.70 & \\
\hline 10341 & -0.931 & 1.130 & 4382 & 2.496 & 1.39 & 4399 & 2.492 & 99.97 & 102.53 & \\
\hline 10571 & -1.214 & 1.210 & 4274 & 2.640 & 1.21 & 4252 & 2.646 & 106.20 & 112.98 & \\
\hline 10681 & -2.045 & 1.496 & 3931 & 3.114 & 0.59 & 4005 & 3.074 & 97.07 & & \\
\hline 13575 & -0.204 & 1.009 & 4555 & 2.164 & 1.79 & 4569 & 2.161 & & 95.33 & \\
\hline 13983 & 0.350 & 0.867 & 4777 & 1.899 & 2.14 & 4826 & 1.890 & & 110.07 & 109.00 \\
\hline 30523 & -0.101 & 0.949 & 4646 & 2.104 & 1.89 & 4733 & 2.087 & & 102.23 & \\
\hline 30763 & -0.984 & 1.101 & 4422 & 2.507 & 1.40 & 4547 & 2.478 & 94.10 & & \\
\hline 30900 & -0.506 & 1.015 & 4546 & 2.287 & 1.67 & 4666 & 2.262 & 114.32 & 119.94 & \\
\hline 30927 & -2.074 & 1.479 & 3950 & 3.115 & 0.59 & 4138 & 3.029 & 86.78 & & \\
\hline 31851 & -0.369 & 0.979 & 4600 & 2.220 & 1.75 & 4731 & 2.195 & & 93.05 & \\
\hline 32398 & -0.903 & 1.097 & 4427 & 2.473 & 1.43 & 4494 & 2.457 & 94.40 & & \\
\hline 32469 & -1.550 & 1.225 & 4255 & 2.780 & 1.06 & 4379 & 2.744 & & 94.15 & \\
\hline 32685 & 0.066 & 0.918 & 4695 & 2.028 & 1.98 & 4788 & 2.010 & & 87.17 & 94.52 \\
\hline 32862 & -0.424 & 0.993 & 4579 & 2.247 & 1.72 & 4525 & 2.258 & 98.57 & 105.16 & \\
\hline 32909 & -0.568 & 1.030 & 4524 & 2.316 & 1.63 & 4572 & 2.306 & & 90.43 & \\
\hline 32924 & -0.737 & 1.065 & 4473 & 2.396 & 1.53 & 4538 & 2.381 & & 101.03 & \\
\hline 33452 & -0.420 & 1.015 & 4546 & 2.252 & 1.70 & 4671 & 2.226 & 92.79 & & \\
\hline 33918 & -1.259 & 1.225 & 4255 & 2.664 & 1.17 & 4312 & 2.647 & 117.58 & 119.80 & \\
\hline 34008 & -0.471 & 1.007 & 4558 & 2.270 & 1.69 & 4670 & 2.247 & 102.73 & & \\
\hline 34013 & 0.854 & 0.835 & 4830 & 1.688 & 2.37 & 5110 & 1.645 & & & 101.49 \\
\hline 34634 & -0.229 & 1.013 & 4549 & 2.175 & 1.78 & 4627 & 2.159 & & & \\
\hline 35265 & -0.269 & 1.043 & 4505 & 2.201 & 1.74 & 4547 & 2.192 & & 98.95 & \\
\hline 37496 & -0.719 & 1.035 & 4516 & 2.378 & 1.56 & 4547 & 2.372 & 109.69 & 109.75 & \\
\hline 37505 & -0.807 & 1.055 & 4487 & 2.420 & 1.51 & 4504 & 2.416 & 96.87 & & \\
\hline 37872 & -1.940 & 1.464 & 3967 & 3.052 & 0.66 & 4015 & 3.028 & 104.96 & & 104.94 \\
\hline 37998 & -1.357 & 1.185 & 4307 & 2.687 & 1.17 & 4317 & 2.684 & 90.72 & 94.27 & \\
\hline 38228 & -0.609 & 1.010 & 4553 & 2.326 & 1.63 & 4588 & 2.319 & & 101.96 & \\
\hline 38244 & -0.397 & 0.965 & 4621 & 2.227 & 1.75 & 4659 & 2.220 & & 106.87 & \\
\hline 38660 & -1.300 & 1.162 & 4338 & 2.656 & 1.22 & 4332 & 2.657 & 96.62 & & \\
\hline 38967 & -0.338 & 0.943 & 4655 & 2.197 & 1.80 & 4717 & 2.185 & & 98.44 & \\
\hline 39060 & -1.173 & 1.156 & 4346 & 2.602 & 1.27 & 4357 & 2.600 & & 113.95 & \\
\hline 40196 & -1.020 & 1.104 & 4418 & 2.522 & 1.38 & 4413 & 2.524 & 97.76 & & \\
\hline 40983 & -1.230 & 1.143 & 4364 & 2.620 & 1.26 & 4364 & 2.620 & 106.19 & 118.90 & \\
\hline 41008 & -0.401 & 0.930 & 4676 & 2.218 & 1.78 & 4684 & 2.216 & & 94.62 & \\
\hline 41828 & -1.069 & 1.121 & 4394 & 2.548 & 1.35 & 4409 & 2.544 & & 100.96 & \\
\hline 41969 & -1.144 & 1.121 & 4394 & 2.578 & 1.32 & 4377 & 2.583 & 79.00 & 88.81 & \\
\hline 42073 & -1.299 & 1.191 & 4299 & 2.666 & 1.19 & 4268 & 2.676 & & 101.15 & \\
\hline 42165 & 0.797 & 0.823 & 4851 & 1.707 & 2.36 & 4906 & 1.698 & & 108.81 & \\
\hline 42789 & -0.223 & 0.889 & 4741 & 2.134 & 1.89 & 4813 & 2.122 & & 115.68 & \\
\hline 42886 & 0.331 & 0.878 & 4759 & 1.910 & 2.12 & 4791 & 1.904 & & 109.36 & 114.20 \\
\hline 42996 & -0.164 & 0.906 & 4714 & 2.116 & 1.90 & 4769 & 2.106 & & 91.47 & \\
\hline 43041 & -1.867 & 1.406 & 4033 & 2.990 & 0.75 & 4035 & 2.989 & 112.22 & 109.48 & \\
\hline
\end{tabular}


Table 2. continued.

\begin{tabular}{|c|c|c|c|c|c|c|c|c|c|c|}
\hline ID No. & $\overline{M_{V}}$ & $\overline{(B-V)_{0}}$ & $\begin{array}{c}T_{\text {eff }} \\
(B-V)\end{array}$ & $\begin{array}{c}\log L \\
(B-V)\end{array}$ & $\begin{array}{c}\log g \\
(B-V)\end{array}$ & $\begin{array}{c}T_{\text {eff }} \\
(V-K)\end{array}$ & $\begin{array}{c}\log L \\
(V-K)\end{array}$ & $\begin{array}{r}R V_{02} \\
\mathrm{~km} \mathrm{~s}^{-1}\end{array}$ & $\begin{array}{r}R V_{12} \\
\mathrm{~km} \mathrm{~s}^{-1}\end{array}$ & $\begin{array}{r}R V_{\mathrm{U}} \\
\mathrm{km} \mathrm{s}^{-1}\end{array}$ \\
\hline 43217 & 0.850 & 0.811 & 4871 & 1.683 & 2.39 & 4916 & 1.676 & & & 103.23 \\
\hline 43247 & -0.804 & 1.016 & 4544 & 2.406 & 1.55 & 4463 & 2.425 & 106.76 & 107.76 & \\
\hline 43281 & -0.543 & 1.003 & 4564 & 2.298 & 1.66 & & & 95.23 & & \\
\hline 43561 & -2.267 & 1.619 & 3801 & 3.294 & 0.35 & 3872 & 3.240 & 92.69 & & \\
\hline 43794 & -1.036 & 1.087 & 4442 & 2.523 & 1.39 & 4431 & 2.526 & 97.95 & 101.91 & \\
\hline 43822 & -0.990 & 1.111 & 4408 & 2.513 & 1.39 & 4411 & 2.512 & 88.92 & 99.96 & \\
\hline 44573 & -0.501 & 0.976 & 4605 & 2.272 & 1.70 & 4516 & 2.291 & 88.63 & & \\
\hline 44665 & -1.345 & 1.184 & 4308 & 2.682 & 1.18 & 4310 & 2.681 & 91.94 & 93.67 & \\
\hline 44716 & -0.691 & 1.004 & 4562 & 2.357 & 1.60 & 4539 & 2.362 & 99.30 & & \\
\hline 44984 & -1.055 & 1.100 & 4423 & 2.535 & 1.37 & 4405 & 2.540 & 86.65 & 82.96 & \\
\hline 45162 & -2.327 & 1.689 & 3731 & 3.386 & 0.22 & 3810 & 3.310 & 112.90 & & \\
\hline 45443 & -1.016 & 1.091 & 4436 & 2.516 & 1.39 & 4425 & 2.519 & 99.23 & 96.10 & \\
\hline 45840 & -1.473 & 1.215 & 4268 & 2.745 & 1.10 & 4220 & 2.760 & 95.49 & 100.90 & \\
\hline 46041 & -0.623 & 0.979 & 4600 & 2.322 & 1.65 & 4568 & 2.329 & 93.63 & 103.22 & \\
\hline 46099 & -1.849 & 1.414 & 4024 & 2.987 & 0.75 & 4032 & 2.983 & 102.55 & & 103.48 \\
\hline 46367 & -0.758 & 1.002 & 4565 & 2.383 & 1.58 & 4383 & 2.427 & & 95.19 & \\
\hline 46422 & -2.214 & 1.578 & 3843 & 3.239 & 0.42 & & & & & 93.50 \\
\hline 46580 & -1.900 & 1.382 & 4061 & 2.991 & 0.77 & & & 102.34 & & 103.12 \\
\hline 46663 & -1.039 & 1.089 & 4439 & 2.525 & 1.39 & 4474 & 2.516 & & 97.26 & \\
\hline 46726 & -1.215 & 1.145 & 4361 & 2.615 & 1.27 & 4360 & 2.616 & 90.03 & & \\
\hline 46868 & -0.838 & 1.031 & 4522 & 2.425 & 1.52 & & & & 110.38 & \\
\hline 46924 & -0.764 & 1.050 & 4495 & 2.402 & 1.53 & & & 96.36 & & \\
\hline 47031 & -1.409 & 1.204 & 4282 & 2.715 & 1.13 & 4076 & 2.788 & 95.43 & & \\
\hline 47145 & -1.943 & 1.382 & 4061 & 3.008 & 0.75 & 4051 & 3.012 & 66.67 & 75.97 & \\
\hline 47421 & -1.415 & 1.176 & 4319 & 2.707 & 1.16 & 4288 & 2.716 & 74.65 & 69.43 & \\
\hline 47452 & -1.813 & 1.860 & 3570 & 3.457 & 0.08 & 3737 & 3.174 & 101.28 & & \\
\hline 47606 & -2.154 & 1.601 & 3819 & 3.233 & 0.42 & 3839 & 3.218 & 96.25 & & 97.77 \\
\hline 48011 & -1.193 & 1.101 & 4422 & 2.591 & 1.31 & 4375 & 2.603 & 98.87 & 101.85 & \\
\hline 48060 & -2.034 & 1.480 & 3949 & 3.099 & 0.61 & 3969 & 3.088 & 96.03 & 91.58 & \\
\hline 48128 & -1.106 & 1.121 & 4394 & 2.563 & 1.33 & 4368 & 2.570 & 96.16 & 101.22 & \\
\hline 48424 & -1.138 & 1.100 & 4423 & 2.568 & 1.34 & 3649 & 3.020 & 109.01 & & \\
\hline 48609 & -2.175 & 1.650 & 3769 & 3.286 & 0.34 & 3846 & 3.221 & & & 85.20 \\
\hline 48889 & -2.249 & 1.578 & 3843 & 3.253 & 0.41 & 3943 & 3.188 & 114.00 & & 116.29 \\
\hline 49509 & -1.669 & 1.288 & 4175 & 2.854 & 0.95 & 4164 & 2.858 & & 106.58 & \\
\hline 49680 & -2.023 & 1.469 & 3961 & 3.088 & 0.63 & 3952 & 3.094 & & 83.64 & \\
\hline 49743 & -0.422 & 0.965 & 4621 & 2.237 & 1.74 & 4665 & 2.228 & 107.18 & 112.48 & \\
\hline 49753 & -1.240 & 1.099 & 4425 & 2.609 & 1.30 & 4298 & 2.643 & 112.04 & & \\
\hline 49942 & -1.854 & 1.444 & 3989 & 3.006 & 0.72 & 4023 & 2.990 & & 118.85 & \\
\hline 50119 & -1.704 & 1.319 & 4136 & 2.882 & 0.91 & 4166 & 2.871 & 100.83 & & 101.56 \\
\hline 50371 & -1.750 & 1.404 & 4035 & 2.942 & 0.80 & 4054 & 2.934 & & 104.36 & \\
\hline 50561 & -0.756 & 1.066 & 4471 & 2.404 & 1.52 & 4429 & 2.414 & 92.71 & & \\
\hline 50681 & -1.930 & 1.423 & 4013 & 3.025 & 0.71 & 4114 & 2.981 & 115.24 & & \\
\hline 50761 & -2.200 & 1.784 & 3639 & 3.462 & 0.10 & 3756 & 3.309 & 103.34 & & 99.80 \\
\hline 50861 & -2.034 & 1.425 & 4011 & 3.067 & 0.67 & 4039 & 3.054 & 108.07 & 110.60 & \\
\hline 50866 & -0.956 & 1.087 & 4442 & 2.491 & 1.42 & 4432 & 2.493 & & 95.50 & \\
\hline 50910 & -0.606 & 1.030 & 4524 & 2.332 & 1.61 & 4577 & 2.320 & & 98.33 & \\
\hline 51416 & -0.645 & 1.023 & 4534 & 2.345 & 1.60 & 4546 & 2.342 & 87.40 & 90.40 & \\
\hline 51454 & -2.144 & 1.567 & 3855 & 3.202 & 0.46 & 3893 & 3.177 & 82.79 & & 83.35 \\
\hline 51499 & -2.155 & 1.502 & 3925 & 3.162 & 0.54 & 3960 & 3.142 & 108.71 & & 109.18 \\
\hline 51515 & -1.377 & 1.379 & 4065 & 2.780 & 0.98 & 4154 & 2.745 & 104.52 & 106.77 & \\
\hline 51646 & -0.130 & 0.870 & 4772 & 2.092 & 1.95 & 4886 & 2.072 & & 99.69 & \\
\hline 51871 & -2.048 & 1.547 & 3876 & 3.149 & 0.53 & 3970 & 3.094 & & 93.84 & \\
\hline 51930 & -1.522 & 1.243 & 4232 & 2.776 & 1.05 & 4214 & 2.782 & 90.34 & & \\
\hline 51963 & -1.391 & 1.233 & 4244 & 2.720 & 1.11 & 4242 & 2.720 & 100.59 & 102.54 & \\
\hline 51983 & -2.116 & 1.634 & 3786 & 3.247 & 0.39 & 3855 & 3.191 & & & 101.48 \\
\hline 52006 & -1.268 & 1.111 & 4408 & 2.624 & 1.27 & 4397 & 2.627 & 108.92 & 109.48 & \\
\hline
\end{tabular}


Table 2. continued.

\begin{tabular}{cccccccccc}
\hline \hline ID No. & $M_{V}$ & $(B-V)_{0}$ & $\begin{array}{c}T_{\text {eff }} \\
(B-V)\end{array}$ & $\begin{array}{c}\log L \\
(B-V)\end{array}$ & $\begin{array}{c}\log g \\
(B-V)\end{array}$ & $\begin{array}{c}T_{\text {eff }} \\
(V-K)\end{array}$ & $\begin{array}{c}\log L \\
(V-K)\end{array}$ & $\begin{array}{r}R V_{02} \\
\mathrm{~km} \mathrm{~s}^{-1}\end{array}$ & $\begin{array}{r}R V_{12} \\
\mathrm{~km} \mathrm{~s}^{-1}\end{array}$ \\
\hline 52048 & -1.331 & 1.166 & 4333 & 2.670 & 1.20 & 4294 & 2.681 & 75.18 & \\
$\mathrm{~km} \mathrm{~s}^{-1}$
\end{tabular}

\section{Results and discussion}

\subsection{Ho line}

\subsubsection{Emission}

We show in Fig. 4 the $\mathrm{H} \alpha$ line profiles for the 20 stars observed with UVES. One can see that most of the stars brighter than $V=14$ show clear evidence of emission wings, mostly on the blue side, but also on both sides, whereas the fainter ones do not show such features. We note that weak emission from the wings could escape detection as it would be compensated by the absorption, the net result being a narrower absorption profile. In order to reveal all possible evidence of emission, after careful visual inspection we have arbitrarily identified a "template" star with no obvious $\mathrm{H} \alpha$ emission and a fairly symmetric profile. This star is \#53390 $(V=14.67$, $M_{V}=-0.9, T_{\text {eff }} \sim 4400 \mathrm{~K}$ and $\left.\log L / L_{\odot} \sim 2.5\right)$. We have then subtracted the $\mathrm{H} \alpha$ profile of \#53390 from all stars in our UVES sample brighter than $V=14$ after normalizing the intensities to the continuum and shifting in wavelength to superpose the bisector of the $\mathrm{H} \alpha$ profile at the level of half maximum intensity. We have applied this procedure only to the stars in our UVES sample brighter than $V=14.5$ because the fainter ones become progressively hotter and the $\mathrm{H} \alpha$ profiles have a different shape, besides showing no indication whatsoever of emission wings. However, the possible dependence of the $\mathrm{H} \alpha$ line shape on temperature might introduce spurious features in the subtracted profiles. In order to test for this effect, we have calculated the theoretical $\mathrm{H} \alpha$ profiles for our stars using Kurucz model atmospheres (with no chromosphere) and the individual values of temperature and gravity estimated from the $(B-V)$ colors that are listed in Table 2. In Fig. 5 we show the difference of the observed spectra (star-\#53390) and, for comparison, the difference of the corresponding theoretical profiles. Clearly the observed profiles are quite different from the theoretical ones as predicted just from the variations of temperature and gravity along the RGB. In particular: i) in all stars the absorption at the center of the line is larger than in the template star, contrary to the theoretical expectations. This suggests the presence of a thicker atmosphere (i.e. an excess of material) and/or a higher temperature in the external regions (i.e. a chromosphere). This absorption is often blue-shifted, as clearly appears in the stars $48889,51499,51454,51983,37872,46580$ and 56032. ii) Whereas the red emission wing could be due to the subtraction procedure in some case (see e.g. the stars 48609 or 56032), the blue emission wing seems real in all cases (except perhaps for star 51983). We note that the stars 48889 and 46580, that do not show blue emission, do however show an asymmetric blue-shifted absorption core like star 51983. From Fig. 5, therefore, it appears that 10 out of 11 stars brighter than $\log L / L_{\odot} \sim 2.9$ (i.e. more than $90 \%$ ) show $\mathrm{H} \alpha$ emission wings. The faintest stars of this group, \#50119 and 56032, have $\log L / L_{\odot}=2.87-2.88$; however we cannot take this value as a threshold for the onset of emission wings, since there is a gap of about half a magnitude in our UVES sample and the monitoring is not continuos in luminosity.

We have applied a similar procedure to the stars with $\log L / L_{\odot}>2.5$ that were observed with GIRAFFE/MEDUSA and setup HR14. Unlike for the UVES stars, we did not attempt to estimate the velocities of the emission peaks with respect to the bisector of the $\mathrm{H} \alpha$ absorption profile, because of the shaky wavelength calibration. Instead, we have shifted the spectra to $R V=0$ using nearby photospheric lines. In this case the template spectrum used for subtraction is the average of 


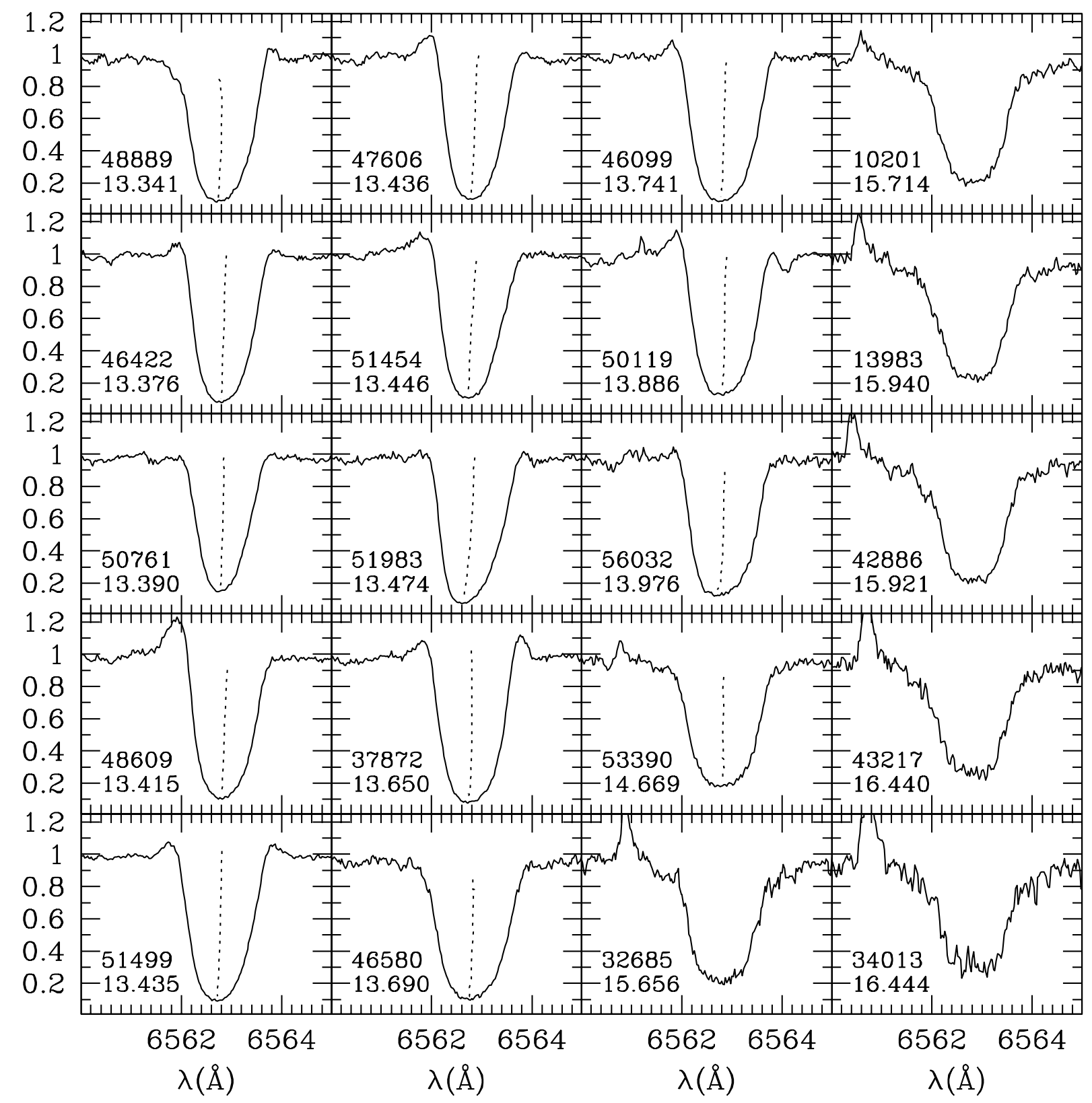

Fig. 4. Normalised parts of spectra containing the $\mathrm{H} \alpha$ line for the 20 stars observed with UVES, shifted to $R V=0$. We also plot the line bisectors for the brighter stars. Note that spectra for stars fainter than $V=14.5$ show the sky $\mathrm{H} \alpha$ line in emission on the blue side of the stellar $\mathrm{H} \alpha$ line.

three spectra, i.e. those of stars \#8603, 10571 and 43822, that have temperatures in the range $T_{\text {eff }}=4250-4400 \mathrm{~K}$ and luminosities in the range $\log L / L_{\odot}=2.50-2.65$. These parameters are quite similar to those of star \#53390 that was selected as a template for the UVES spectra. The much larger number of stars observed with GIRAFFE gives us a more detailed monitoring of the $\mathrm{H} \alpha$ line behaviour as a function of luminosity, but of course the GIRAFFE lower resolution might more easily hide weak $\mathrm{H} \alpha$ emission wings in the strong $\mathrm{H} \alpha$ absorption line.

From the GIRAFFE sample we estimate that the fractions of stars showing some evidence of $\mathrm{H} \alpha$ emission are approximately as indicated in parenthesis, in the luminosity intervals $\log L / L_{\odot}=2.5-2.6$ (30\%), 2.6-2.7 (73\%), 2.7-2.8 (86\%),
$2.8-2.9(100 \%)$ and $>2.9(100 \%)$. If we include also the UVES results, in the interval $\log L / L_{\odot}>2.9$ the emission detection frequency would be $\sim 95 \%$, and $\sim 94 \%$ for $\log L / L_{\odot}>2.7$.

A comparison with previous studies must take into account that the various sets of results were obtained with different procedures and with spectra of different resolution. Our procedure of subtracting an $\mathrm{H} \alpha$ template, i.e. a supposedly pure absorption profile, was not chosen by anybody else before. In principle it should allow to detect emission features that might have gone unnoticed had this subtraction not been performed, therefore we would expect a higher detection rate because of this. On the other hand, we compare our UVES and GIRAFFE data, with spectral resolution of $\sim 7$ and $10 \mathrm{~km}^{-1}$ respectively, with the major statistical studies on $\mathrm{H} \alpha$ emission in metal-poor red 


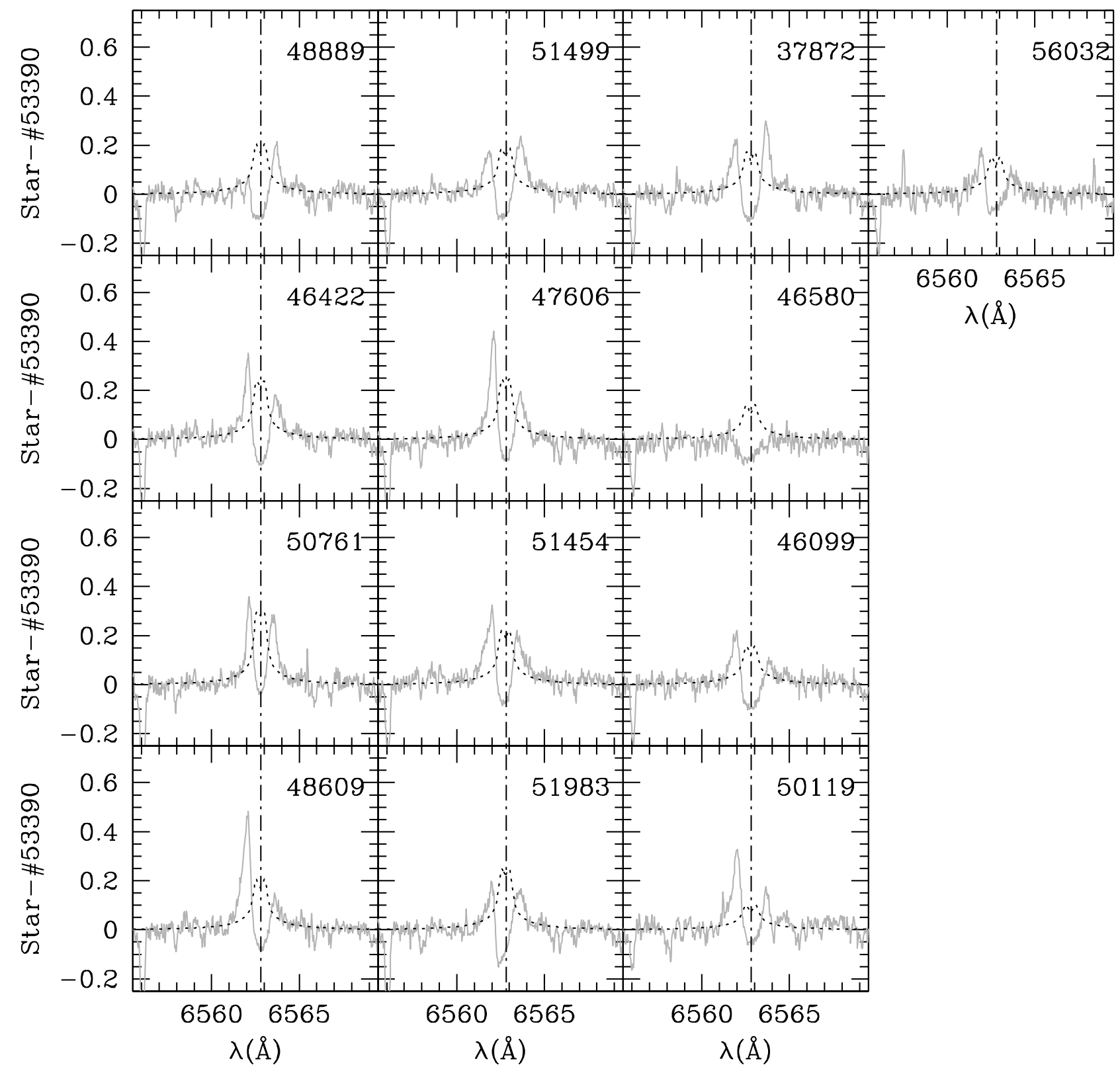

Fig. 5. $\mathrm{H} \alpha$ lines after subtraction of the template profile from star $\# 53390$, for the 13 stars observed with UVES and brighter than $V=$ $14.0\left(\log L / L_{\odot}=2.88\right)$. The solid grey lines show the differences of the observed profiles, the dotted lines show the differences of the corresponding theoretical profiles.

giant stars, namely Cacciari \& Freeman (1983, 143 RGB stars in globular clusters, res. $\sim 30 \mathrm{~km} \mathrm{~s}^{-1}$ ), Gratton et al. (1984, 113 RGB stars in globular clusters, res. $\left.\sim 15 \mathrm{~km} \mathrm{~s}^{-1}\right)$, Smith \& Dupree (1988, 52 metal-poor field red giants, res. $\sim 7 \mathrm{~km} \mathrm{~s}^{-1}$ ), and Lyons et al. (1996, 62 RGB stars in globular clusters, res. $\sim 5 \mathrm{~km} \mathrm{~s}^{-1}$ ). A poorer resolution makes the detection of emission features more difficult. As discussed also by Lyons et al. (1996), $\mathrm{H} \alpha$ emission was detected by Cacciari \& Freeman (1983) only among stars brighter than $\log L / L_{\odot}=2.9$ in a proportion of $\sim 33 \%$, by Gratton et al. (1984) among stars brighter than $\log L / L_{\odot}=2.7$ in a proportion of $\sim 61 \%$, by Smith \& Dupree (1988) above the threshold of $M_{V}=-1.7$ (i.e. $\log L / L_{\odot} \sim 2.9$ ) in a proportion of $\sim 77 \%$ ( $\leq 50 \%$ if reported to the threshold of $\log L / L_{\odot}=2.7$ ), and finally by
Lyons et al. (1996) above the threshold of $\log L / L_{\odot}=2.7$ in a proportion of $\sim 80 \%$.

Our detection limit for $\mathrm{H} \alpha$ emission is $\log L / L_{\odot} \sim 2.9$ from the UVES stars with a proportion of $\sim 91 \%$; from the GIRAFFE stars our detection threshold is $\log L / L_{\odot} \sim 2.5$ with a proportion of $\sim 72 \%$. If we report these results to the threshold value of $\log L / L_{\odot}=2.7$ for the sake of comparison, and consider both UVES and GIRAFFE stars, then $~ 94 \%$ of the stars brighter than this value exhibit $\mathrm{H} \alpha$ emission wings.

Finally, we note that time variability of $\mathrm{H} \alpha$ emission would contribute to underestimate the frequency of $\mathrm{H} \alpha$ emitting stars. The present set of data, however, does not allow us to monitor for possible variability of $\mathrm{H} \alpha$ emission features. 
Table 3. Parameters of the $\mathrm{H} \alpha$ line for the stars observed with UVES. Columns 2-3 refer to the absorption core, Cols. 4-8 refer to the emission features. The parameter $B / R$ is the intensity ratio of the Blue and Red emission wings after subtraction of the template spectrum.

\begin{tabular}{rrrrrrrr}
\hline \hline ID No. & $\begin{array}{r}\text { Centre } \\
\text { Sh. }\end{array}$ & $\begin{array}{r}\text { Core } \\
\text { Sh. }\end{array}$ & $B / R$ & $\begin{array}{r}\text { Blue } \\
\text { Pk. }\end{array}$ & $\begin{array}{r}\text { Blue } \\
\text { Ter. }\end{array}$ & $\begin{array}{r}\text { Red } \\
\text { Pk. }\end{array}$ & $\begin{array}{r}\text { Red } \\
\text { Ter. }\end{array}$ \\
\hline 10201 & -1.20 & -0.76 & & & & & \\
13983 & -0.31 & 0.60 & & & & & \\
32685 & 1.07 & -0.76 & & & & & \\
34013 & 2.43 & 2.43 & & & & & \\
37872 & -0.76 & -2.57 & $<1$ & -44.3 & -79.3 & 39.8 & 57.7 \\
42886 & 0.60 & 0.60 & & & & & \\
43217 & -0.31 & 1.99 & & & & & \\
46099 & 1.52 & -1.20 & $>1$ & -42.3 & -68.8 & 49.9 & 66.4 \\
46422 & 1.52 & -0.76 & $>1$ & -35.7 & -77.5 & 41.1 & 79.2 \\
46580 & 0.16 & -2.57 & & & & & \\
47606 & 2.43 & -0.31 & $>1$ & -34.6 & -92.2 & 38.3 & 66.4 \\
48609 & 1.99 & -0.31 & $>1$ & -39.1 & -86.7 & 38.7 & 65.5 \\
48889 & -0.76 & -3.48 & $<1$ & -33.9 & -42.4 & 39.6 & 61.8 \\
50119 & 1.99 & -0.76 & $>1$ & -39.6 & -86.7 & 39.3 & 57.3 \\
50761 & 0.60 & -1.20 & $\sim 1$ & -30.9 & -67.0 & 31.9 & 52.8 \\
51454 & -1.20 & -3.95 & $>1$ & -43.3 & -83.0 & 35.3 & 75.5 \\
51499 & -1.65 & -4.39 & $<1$ & -47.4 & -85.7 & 39.3 & 79.2 \\
51983 & -1.20 & -8.05 & $\sim 1$ & -41.6 & -83.0 & 35.2 & 55.9 \\
53390 & 0.60 & -0.76 & & & & & \\
56032 & 0.60 & -4.39 & $>1$ & -38.5 & -60.7 & 38.3 & 50.8 \\
\hline
\end{tabular}

Notes:

i) All radial velocities (centre and core shifts, and velocities of emission peaks and terminal emission profiles) are given in $\mathrm{km} \mathrm{s}^{-1}$. Typical errors of individual measures are $\pm 1 \mathrm{~km} \mathrm{~s}^{-1}$.

ii) The centre and core shifts are relative to the rest position of $\mathrm{H} \alpha$, while the velocities of the emission peaks and terminal emission profiles are relative to the $\mathrm{H} \alpha$ line centre (bisector at half maximum).

\subsection{2. $\mathrm{H} \alpha$ emission: Mass loss or chromospheric activity?}

The nature of the $\mathrm{H} \alpha$ emission is controversial. Early studies (cf. Cohen 1976; Mallia \& Pagel 1978; Cacciari \& Freeman 1983; Gratton et al. 1984) assumed that it could be taken as evidence of extended matter around the star hence mass loss could be inferred. However, Dupree et al. (1984) argued that $\mathrm{H} \alpha$ emission could form naturally in a static chromosphere, or it could be enhanced by hydrodynamic processes related to pulsation (Dupree et al. 1994), with no need to invoke mass loss (cf. also Reimers 1981). We discuss here briefly these two hypotheses.

In the hypothesis that the $\mathrm{H} \alpha$ emission is due to circumstellar material, one could compare the observed $E W$ of the emission components with "theoretical" predictions. These predictions are obtained by the use of two different relations, one by Cohen (1976) that gives the mass loss rate as:

$\dot{M}=2.4 \times 10^{-11} V_{\exp } R_{*}\left(R_{\mathrm{s}} W_{\lambda}\right)^{1 / 2} \times \mathrm{e}^{\left(-1.1 / T_{4}\right)} M_{\odot} \mathrm{yr}^{-1}$

where it is assumed that the emission forms in a shell at constant expansion velocity $V_{\exp }$ (taken as the observed velocity of the blue peaks listed in Table 3$)$ and located at a distance $\left(R_{\mathrm{S}}\right)$ of 2 stellar radii $\left(R_{*}\right)$ from the star, $W_{\lambda}$ is the equivalent width

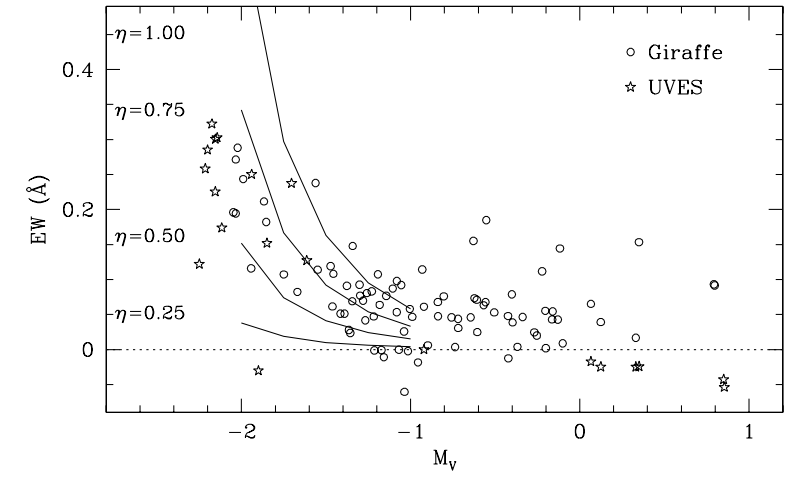

Fig. 6. $E W \mathrm{~s}$ of the emissions on the $\mathrm{H} \alpha$ line wings, as a function of $M_{V}$. The lines show what is expected for different values of $\eta$.

in $\AA$ of the emission components, and $T_{4}$ is the brightness temperature of the star in units of $10^{4} \mathrm{~K}$.

The other relation, by Reimers (1975), gives the mass loss rate as:

$\dot{M}=-4 \times 10^{-13} \eta L / g R$

where all quantities are in solar units and $\eta$ is an empirical scaling factor that can reproduce reasonably well the HB morphology of globular clusters if allowed to vary in the range from 0.25 to 1 .

By imposing that these two parameterizations yield the same value of mass loss rate, and using parameter values that can be derived from the observations or assumed under reasonable assumptions, one can then derive "theoretical" values for the $E W$ 's of the emission components, to be compared with the observed ones. Figure 6 shows a plot of the $E W$ of the emission components, both for stars observed with UVES (starred symbols) and with GIRAFFE (open circles). A good fit of the estimated and observed $E W \mathrm{~s}$ can be obtained for a value of $\eta \sim$ 0.5 . This result does not mean that we are indeed observing mass loss via the $\mathrm{H} \alpha$ emission wings, but only that this possibility is consistent with the admittedly very approximate and uncertain parameterizations available so far.

A difficulty with the Cohen circumstellar model, where the emission region is detached from the stellar atmosphere, is that it does not predict correctly the residual intensity at the center of the $\mathrm{H} \alpha$ absorption. According to this model, in fact, the residual intensity should be larger (if some emission occurs at the stellar radial velocity), or at least equal to the undisturbed photospheric absorption. Therefore, we would expect that $\mathrm{H} \alpha$ should be weaker in cooler stars and the residual intensity at the line center should increase with decreasing temperature. However, the opposite holds, as shown in Fig. 7. The strong absorption at the center of $\mathrm{H} \alpha$ could be justified by assuming that the circumstellar material is disposed like a rotating torus around the star, but this explanation appears unjustified for single stars and it does not support mass loss.

The other hypothesis we are investigating is that the $\mathrm{H} \alpha$ emission is due to chromospheric activity. The effects of a chromosphere on the profile of $\mathrm{H} \alpha$ depend on the amount of material in the chromosphere itself. When the chromosphere is optically thin, the emission shows up at the center of the line, as it is the case for active (Population I) subgiants 


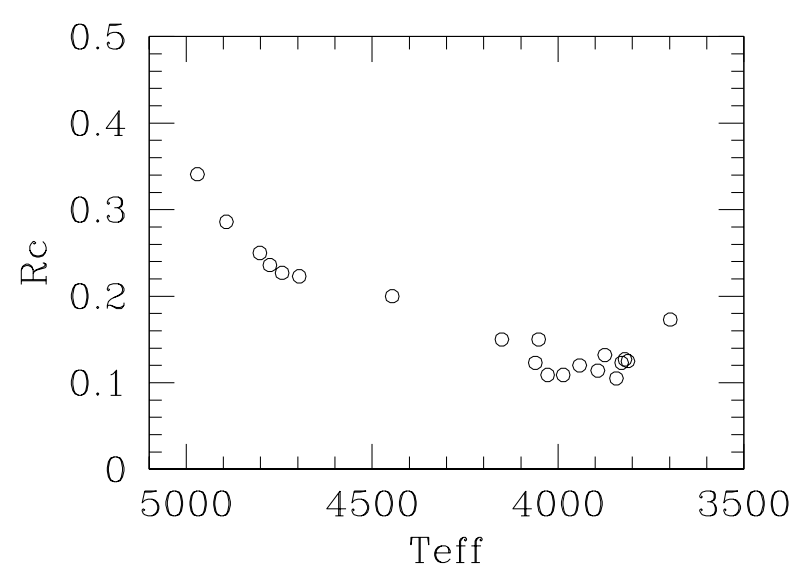

Fig. 7. Central residual intensity $R \mathrm{c}$ of the $\mathrm{H} \alpha$ line as a function of temperature, for the UVES stars only.

(Pasquini \& Pallavicini 1991). In this case the emission causes a filling of the core of the line - its width is almost unaffected being essentially determined by thermal broadening. However, when the chromosphere is optically thick at the center of $\mathrm{H} \alpha$ (this may occur if the lower level of the transition is populated by recombination from a strong enough UV flux in an extended and dense chromosphere), the line profile becomes very different. The mean free path of photons at the $\mathrm{H} \alpha$ wavelength becomes short, and they cannot escape the atmosphere, unless they are slightly shifted in wavelength by anelastic diffusion processes: in this case, they will exit the atmosphere, causing blue- or red-shifted emissions. Detailed calculations of models with extended atmospheres by Dupree et al. (1984) indeed show deep central $\mathrm{H} \alpha$ absorption with residual intensities $\leq 0.1$, as well as blue- and red-shifted emissions. These predictions agree very well with our results for the coolest stars in our sample. We think this gives a strong support to the chromospheric explanation of the $\mathrm{H} \alpha$ emission.

It should be reminded that the presence of an extended chromosphere neither excludes nor requires mass loss. This is shown by the wind model by Dupree et al. (1984), that describes a star with an extended chromosphere and a mass loss of $2 \times 10^{-9} M_{\odot} \mathrm{yr}^{-1}$. In the case of a net outflow motion, slightly red-shifted photons have a higher probability of escape, hence we expect that the red-shifted emission will be stronger than the blue-shifted one (see Fig. 3 of Dupree et al. 1984). We have estimated the relative strengths of the blue (B) and red (R) emission wings in our stars by integrating the flux distribution on the difference spectra in two $1 \AA$-wide bands on the blue and red side of the absorption line (i.e. $\lambda \lambda 6561.3-6562.3$ and 6563.3-6564.3), and further checked by visual inspection. The parameter $B / R$ is reported in Tables 3 and 4 for the stars observed with UVES and GIRAFFE respectively, as $<1$ if the red emission wing is stronger (denoted as red asymmetry), $>1$ if the blue emission wing is stronger (blue asymmetry), and $\sim 1$ if the red and blue wings are of similar strength: blue asymmetry appears to dominate. This confirms and reinforces the observational evidence found by Smith \& Dupree (1988) among metaldeficient field red giants. Since all previous studies that could use repeated observations of the same stars found that both the
Table 4. Stars observed with GIRAFFE and showing $\mathrm{H} \alpha$ emission. The parameter $B / R$ indicates the relative strength of the $\mathrm{H} \alpha$ blue and red emission wings after subtracting the template spectrum.

\begin{tabular}{|c|c|c|}
\hline ID n. & $B / R$ & Comments \\
\hline 7536 & $\sim 1$ & \\
\hline 8739 & $\sim 1$ & \\
\hline 9230 & $>1$ & Strong emission \\
\hline 9724 & $>1$ & Very weak \\
\hline 10265 & $\sim 1$ & \\
\hline 10341 & $\sim 1$ & \\
\hline 32469 & $>1$ & \\
\hline 33918 & $>1$ & \\
\hline 40983 & $>1$ & \\
\hline 41969 & $\sim 1$ & \\
\hline 42073 & $>1$ & \\
\hline 43041 & $>1$ & \\
\hline 44665 & $>1$ & \\
\hline 45840 & $>1$ & \\
\hline 47145 & $>1$ & \\
\hline 47421 & $>1$ & \\
\hline 48011 & $>1$ & \\
\hline 48060 & $>1$ & \\
\hline 48128 & $>1$ & \\
\hline 49509 & $>1$ & \\
\hline 49680 & $>1$ & Strong emission \\
\hline 49942 & $>1$ & \\
\hline 50371 & $>1$ & \\
\hline 50861 & $>1$ & Strong emission \\
\hline 51515 & $>1$ & \\
\hline 51871 & $>1$ & Strong emission \\
\hline 51963 & $>1$ & Very weak \\
\hline 52006 & $\sim 1$ & Very weak \\
\hline 54284 & $\sim 1$ & Weak \\
\hline 54733 & $>1$ & Weak \\
\hline 54789 & $>1$ & \\
\hline 55031 & $>1$ & Strong emission \\
\hline
\end{tabular}

emission-line strengths and the sense of the $B / R$ asymmetry may change with time (on a timescale of months or even days) for any given star, no firm conclusions can be drawn from our data. We can only suggest the possible presence of differential mass motions in the line-forming region. Smith \& Dupree (1988) proposed an alternative explanation of the variability of the emission strength (that we cannot detect but may indeed be present in our stars as well), i.e. fluctuations of the column density or the temperature gradient or both, within the chromosphere, possibly induced by pulsation.

In conclusion, as already noted by Dupree et al. (1984), asymmetries can be altered by episodic events, that may well occur in a (likely) variable chromosphere, as well as from a more complex geometry. Much more information should be obtained by examining the absorption profile, looking for evidence of core blue-shifts. These will be discussed in the next subsection. 


\subsubsection{Shifts and line asymmetry}

In the UVES spectra we derived the position of the $\mathrm{H} \alpha$ absorption line by defining the center of the line as the bisector of the profile at half maximum intensity, and the core of the line as the minimum intensity interpolated by a local parabolic profile.

We have then measured the center and core shifts with respect to the nearby photospheric lines, and assumed that only shifts larger than $3 \sigma$ (i.e. $\sim 2 \mathrm{~km} \mathrm{~s}^{-1}$ ) may be considered significant. Of the 20 stars observed with UVES, 8 show significant coreshifts, 7 of them being blueshifted (indicative of outward motion in the layer of the atmosphere where the $\mathrm{H} \alpha$ line is formed) and 1 of them being redshifted (indicative of a downward flow). The blue-shifted ones are all brighter than $\log L / L_{\odot} \sim 2.88$ and represent $\sim 54 \%$ of the UVES stars brighter than this value; the star with a red-shifted $\mathrm{H} \alpha$ core is about 3 mag fainter. Only two stars show possibly significant centre shifts, both redward: one is the same star that shows also a redshifted core, the other one shows no significant coreshift. There is no apparent correlation between the occurrence of a blue coreshift and the asymmetry of the $\mathrm{H} \alpha$ emission wings: of the 7 stars where a blue coreshift was detected, 2 have $B / R>$ 1, 3 have $B / R<1,1$ has $B / R \sim 1$ and 1 does not show emission wings. Five other stars exhibiting blue asymmetry do not show any significant coreshift. The GIRAFFE spectra have a resolution about a factor 2 worse than the UVES data, and the precision is hardly good enough to reveal coreshifts such as those detected in the UVES spectra, hence we did not try to estimate them.

Lyons et al. (1996), thanks to the higher resolution ( $R=60000)$ of their spectra, were able to detect and measure significant $\mathrm{H} \alpha$ coreshifts in $\sim 50 \%$ of stars brighter than $\log L / L_{\odot} \sim 2.5$. We cannot reach this limit with our UVES data, and GIRAFFE's resolution is not adequate for reliable measures of such small shifts. Therefore it appears that $\log L / L_{\odot} \sim 2.5$ is the threshold for the occurrence of both $\mathrm{H} \alpha$ emission (our present results) and $\mathrm{H} \alpha$ coreshifts (Lyons et al. 1996), at least based on the presently available data.

In all cases the shifts are $\leq 10 \mathrm{~km} \mathrm{~s}^{-1}$, as it was found also by Smith \& Dupree (1988) from a sample of 52 metal-poor field red giants and using echelle spectra of similar resolution.

\section{2. $\mathrm{Na}$ I $\mathrm{D}$ lines}

In addition to the 20 stars that were observed with UVES, 82 more stars were observed with GIRAFFE/MEDUSA and setup HR12. The contributions from the interstellar $\mathrm{Na}$ lines are clearly separated, since the cluster heliocentric mean radial velocity is $\sim 100 \mathrm{~km} \mathrm{~s}^{-1}$.

We have measured the coreshifts of both $\mathrm{Na}$ I $\mathrm{D}_{1}$ and $\mathrm{D}_{2}$ lines with respect to the photospheric LSR, and in Fig. 8 we show the shifts of the $\mathrm{D}_{2}$ line (which are generally larger, i.e. more negative, than those of the $\mathrm{D}_{1}$ line) as a function of magnitude for all our stars. As already commented by Lyons et al. (1996), "this suggests that, in most cases, there is an outward-increasing velocity gradient in the atmosphere of these cluster giants (although often for individual stars there is no

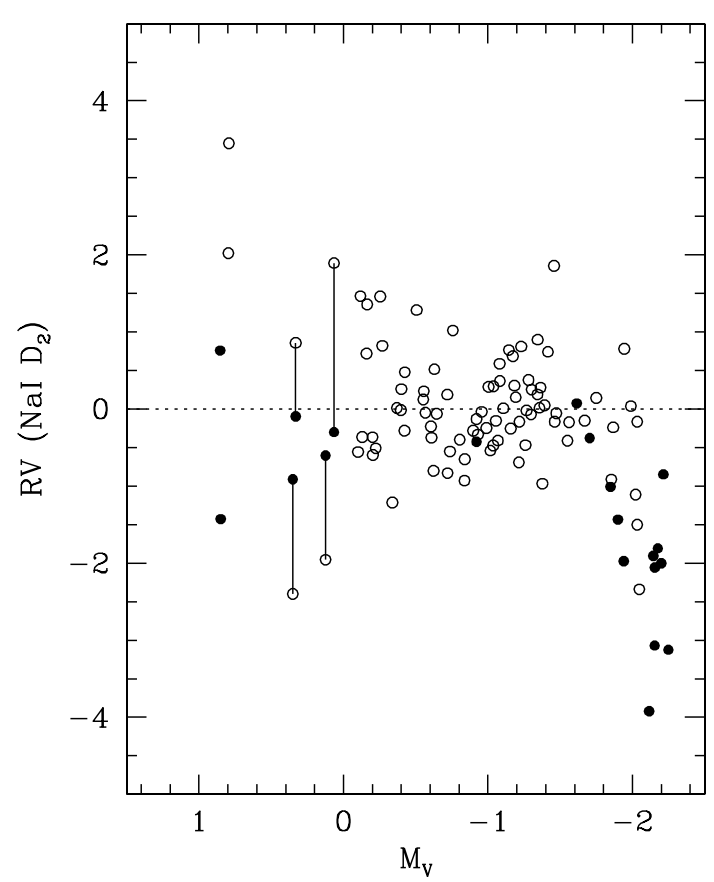

Fig. 8. The coreshifts of the Na $\mathrm{I}_{2}$ lines with respect to the photospheric LSR, as a function of the $V$ magnitude. The filled circles indicate the stars observed with UVES, the open circles indicate the stars observed with GIRAFFE. Four stars have been observed by both, the two measures are connected by a line. Typical errors of the Na I D coreshifts are $\pm 0.6 \mathrm{~km} \mathrm{~s}^{-1}$ for the UVES data and $1.5 \mathrm{~km} \mathrm{~s}^{-1}$ for the GIRAFFE data, except for the faintest stars where the errors are larger.

significant velocity difference between the regions of the atmosphere where the $\mathrm{Na}_{2}$ and $\mathrm{D}_{1}$ line cores are formed)." Typical errors of these measures are $\pm 0.6 \mathrm{~km} \mathrm{~s}^{-1}$ for the UVES data and $\pm 1.5 \mathrm{~km} \mathrm{~s}^{-1}$ for the GIRAFFE data, with slightly worse values for the faintest stars of our sample. We note that, within these errors, we do not detect any significant (i.e. $\geq 3 \sigma$ ) coreshift in stars fainter than $M_{V} \sim-1.8$ (i.e. $\log L / L_{\odot} \geq 2.9$ ). Considering only the UVES data, 8 out of the 11 stars brighter than this value show a significant negative coreshift, in very good agreement with the occurrence of the $\mathrm{H} \alpha$ emission wings.

So, the luminosity limit of $\log L / L_{\odot} \sim 2.9$ for the onset of significant $\mathrm{Na} \mathrm{D}_{2}$ coreshifts is the same as the value found by Lyons et al. (1996), but our detection frequency ( 73\%) is somewhat higher than theirs $(\sim 50 \%)$. Also, our values for the coreshifts of the $\mathrm{D}_{2}$ line reach at most $-4 \mathrm{~km} \mathrm{~s}^{-1}$, whereas Lyons et al. (1996) found up to $\sim-8 \mathrm{~km} \mathrm{~s}^{-1}$ in a few stars of the globular cluster M 13 and one star in M 55. This may be a real effect, or might be at least partly due to the resolution of our spectra that is a factor $\sim 1.3$ lower than Lyons et al.'s.

Equivalent widths of the Na I D lines have been measured for all stars, and the $\mathrm{D}_{2}$ line is the stronger of the $\mathrm{Na}$ I $\mathrm{D}$ pair, hence is formed higher in the atmosphere than the $\mathrm{D}_{1}$ line (as also suggested by the larger velocities of the coreshifts). The values of the individual equivalent widths are not presented here, as they are used elsewhere (Carretta et al. 2003) to perform a detailed $\mathrm{Na}$ abundance analysis.

Lyons et al. (1996) discuss in some detail the relationship between the coreshift and equivalent width of the $\mathrm{Na}_{2}$ line for 
their total sample of 63 stars in 5 globular clusters, and note that significant coreshifts are found only for stars with $E W\left(\mathrm{D}_{2}\right) \geq$ $350 \mathrm{~m} \AA$, with a frequency of about $45 \%$. In all of their clusters the variation of the $E W\left(\mathrm{D}_{2}\right)$ is rather large, except in one cluster (M 55) where all values of the $E W\left(\mathrm{D}_{2}\right)$ clump below $350 \mathrm{~m} \AA$, and no significant coreshifts are detected. This strength threshold, however, does not seem to represent a physical threshold for the onset of mass-motion phenomena, since in the same M 55 stars there is evidence for mass motions via $\mathrm{H} \alpha$ coreshifts and/or asymmetric $\mathrm{H} \alpha$ emission.

It is worth mentioning here that the strength of the $\mathrm{D}_{2}$ line can vary significantly from star to star at any luminosity level due to intrinsic variations of the $\mathrm{Na}$ abundance, as it has been found in our NGC 2808 stars, as well as in other RGB stars previously studied in the globular clusters M 13, M 5, M 15 and M 92 (cf. Carretta et al. 2003 for a discussion). Based on Lyons et al. (1996) results, these variations in $\mathrm{Na}$ abundance within the same cluster RGB stars might lead to miss a significant fraction of $\mathrm{D}_{2}$ coreshifts if the corresponding equivalent width happened to fall below the detection threshold of about $350 \mathrm{~m} \AA$. Therefore, the use of the $\mathrm{Na}_{2}$ line negative coreshifts as indicators of mass motions in the atmospheres of red giants, as good as it may be, could underestimate the real frequency of this phenomenon because of this effect.

If chromospheric activity were at work, one might expect that the entire atmospheric structure is somewhat affected. The onset of $\mathrm{H} \alpha$ emission seems to occur at $\log L / L_{\odot} \sim 2.5$ and $T_{\text {eff }} \sim 4400 \mathrm{~K}$. It is interesting to note that, starting approximately at this value of temperature, the $\mathrm{Na}$ abundances derived by Carretta et al. (2003) for hotter stars tend to be systematically lower, possibly suggesting some degree of line filling. Also the $\mathrm{Fe}$ abundances are slightly smaller, as if the region where these line form were hotter than predicted by the models.

\subsection{Ca II $K$ lines}

The $\mathrm{Ca}$ II $\mathrm{H}$ and $\mathrm{K}$ lines are formed in the chromospheres of cool stars and appear as a deep absorption doublet with a central emission core that may be itself centrally reversed. These lines are often used to identify mass motions and the presence of circumstellar material in luminous stars via their asymmetries (see e.g. Reimers 1977a,b; Dupree 1986), but of course the presence of the chromosphere complicates their interpretation, as well as for the $\mathrm{Na}$ and $\mathrm{H} \alpha$ lines, because of the similarity between the spectral signatures of chromospheres and mass loss.

We have observed 83 stars with the GIRAFFE HR02 setup centered on the $\mathrm{Ca}$ II $\mathrm{H}$ and $\mathrm{K}$ lines: this is the first time that such a large collection of $\mathrm{Ca}$ II $\mathrm{H}$ and $\mathrm{K}$ data for stars belonging to a globular cluster is available. Previous investigations of chromospheric activity in globular cluster giants using Ca II K data were limited to two stars in NGC 6752 (Dupree et al. 1994). Our data show a well defined Ca II reversal in several stars, and confirm the widespread presence of chromospheres among RGB stars of globular clusters.

We display in Fig. 9 the normalised region of the spectra containing the $\mathrm{Ca}$ II $\mathrm{H}$ and $\mathrm{K}$ lines for some of the brightest
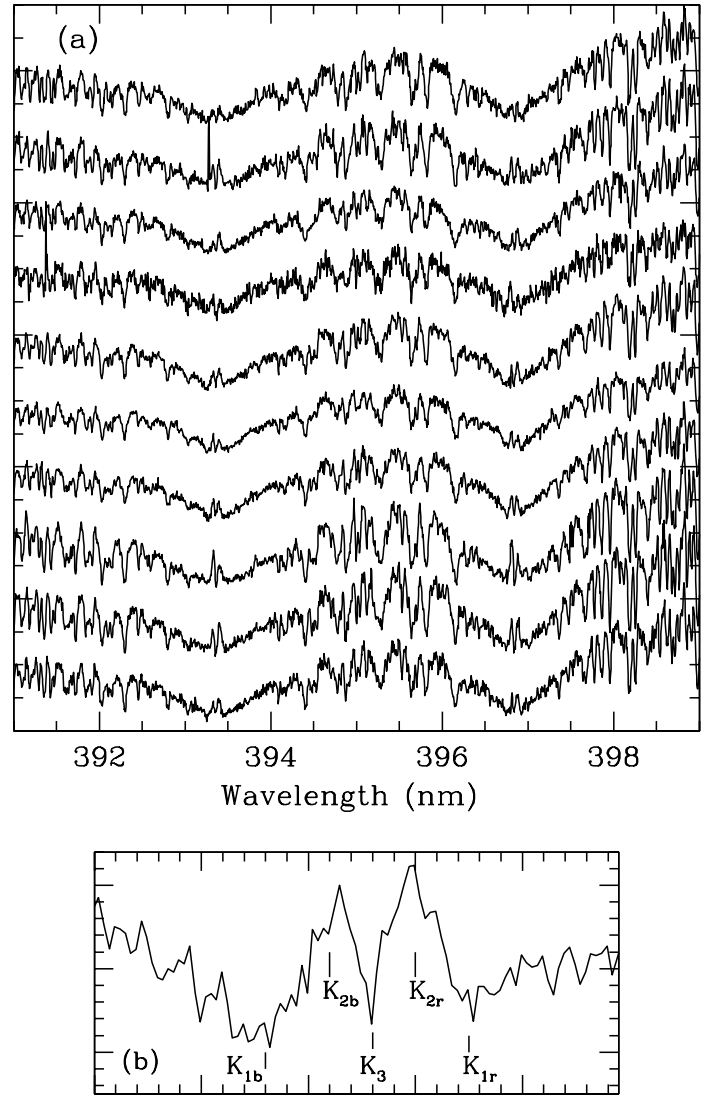

$\begin{array}{llllll} & 393.1 & 393.2 & 393.3 & 393.4 & 393.5\end{array}$

Wavelength $(\mathrm{nm})$

Fig. 9. Parts of spectra containing the $\mathrm{Ca}$ II $\mathrm{H}$ and $\mathrm{K}$ lines, for some of the brightest stars we have observed. The insert shows the zoomed part of the K line for star \#51499.

stars we have observed. The insert shows the bottom of the $\mathrm{K}$ line for star \#51499, zoomed to show the $K_{1}, K_{2}$ and $K_{3}$ components. If differential motions are present in the line-forming region, these peaks may be unequal in intensity and the central line core $\left(K_{3}\right)$ shifted and asymmetric as the line opacity is moved to the blue (expansion) or to the red (contraction). Expansion causes the red emission peak $\left(K_{2 \mathrm{r}}\right)$ to be strenghtened relative to the blue emission peak $\left(K_{2 \mathrm{~b}}\right)$, as clearly seen in Fig. 10.

\subsubsection{Emission frequency and asymmetries}

We have observed 83 stars with the GIRAFFE/MEDUSA HR02 setup; of these, 22 show the central emission and reversal features described above. They are all brighter than $V=$ $14.4\left(\log L / L_{\odot} \sim 2.6\right)$, and represent approximately $50 \%$ of our observed sample in this upper luminosity interval.

For these stars we have measured the relative strength of the two peaks ( $K_{2 \mathrm{~b}}$ and $K_{2 \mathrm{r}}$ ) in the profile of the $K_{2}$ emission reversal, usually denoted by $B / R$ (i.e. the ratio of the intensity of the short-wavelength to the long-wavelength peaks). The $B$ and $R$ intensities were estimated by fitting a Gaussian profile to both $K_{2 \mathrm{~b}}$ and $K_{2 \mathrm{r}}$ components of the emission (see e.g. Dupree $\&$ Smith 1995). Only when the $S / N$ was too poor, the relative 
Table 5. Parameters of the Ca II K line. The $K_{3}$ shifts are measured relative to the nearby photospheric absorption lines.

\begin{tabular}{rccr}
\hline \hline Star ID. & $B / R$ & $\begin{array}{c}\log W \\
\mathrm{~km} \mathrm{~s}^{-1}\end{array}$ & $\begin{array}{r}K_{3} \text { shift } \\
\mathrm{km} \mathrm{s}^{-1}\end{array}$ \\
\hline 9992 & $<1$ & 1.89 & -6.71 \\
10681 & $<1$ & 2.00 & -4.88 \\
30927 & $>1:$ & 1.98 & -1.45 \\
37872 & $<1$ & 1.86 & -5.03 \\
43561 & $>1$ & 1.97 & -5.49 \\
45162 & $<1:$ & 1.99 & -1.30 \\
46099 & $<1$ & 1.93 & -8.47 \\
46580 & $<1$ & 1.89 & -5.03 \\
46726 & $>1$ & 1.83 & +3.36 \\
47031 & $>1$ & 1.80 & -3.97 \\
47606 & $>1:$ & 1.93 & -6.25 \\
48889 & $<1$ & 1.91 & -5.19 \\
50119 & $<1$ & 1.87 & -0.38 \\
50681 & $<1$ & 1.95 & -6.10 \\
50761 & $>1$ & 2.06 & -2.29 \\
51454 & $<1$ & 1.90 & -7.70 \\
51499 & $<1$ & 1.99 & -6.25 \\
51930 & $>1$ & 1.90 & +12.89 \\
52048 & $>1$ & 1.87 & +0.69 \\
53284 & $<1$ & 2.03 & -12.20 \\
56536 & $<1$ & 1.90 & +0.38 \\
56924 & $>1:$ & $1.83:$ & +6.41 \\
\hline & & &
\end{tabular}

intensities were estimated by eye. When outward motions are present in the line-forming region, the intensity ratio $B / R<1$ due to the increased opacity on the short-wavelength side of the line. In our sample, the onset of the $B / R<1$ (red) asymmetry seems to occur at $\log L / L_{\odot} \sim 2.87$, and applies to about $75 \%$ of the stars brighter than this value.

We list in Table 5 these stars and corresponding $B / R$ emission asymmetry.

\subsubsection{Velocity shifts of the $K_{3}$ reversal}

The $K_{3}$ self-reversal in the center of the $K_{2}$ emission line gives a direct measure of the velocity in the chromosphere at the highest point of $\mathrm{Ca}$ line formation. Velocities of the $K_{3}$ reversal have been measured relative to the nearby photospheric absorption lines. Typical uncertainties of these measures amount to $\pm 1.5 \mathrm{~km} \mathrm{~s}^{-1}$, and we may assume again that only shifts larger than $3 \sigma$ (i.e. $\sim 4.5 \mathrm{~km} \mathrm{~s}^{-1}$ ) can be considered significant. The values of the $K_{3}$ velocity shifts are listed in Table 5. They are more frequently negative than positive, mostly negative for the most luminous stars, and less than $15 \mathrm{~km} \mathrm{~s}^{-1}$ for all stars listed in the table. This value is much less than is needed for escape from the photosphere, but mass loss cannot be excluded if a sufficiently high acceleration were attained at large distances from the star, depending on the acceleration mechanism.

Negative $K_{3}$ velocity shifts are taken to indicate that there is an outflow of material in the region of formation of the $K_{3}$ core reversal. We show in Fig. 10a a plot of the $K_{3}$ velocity
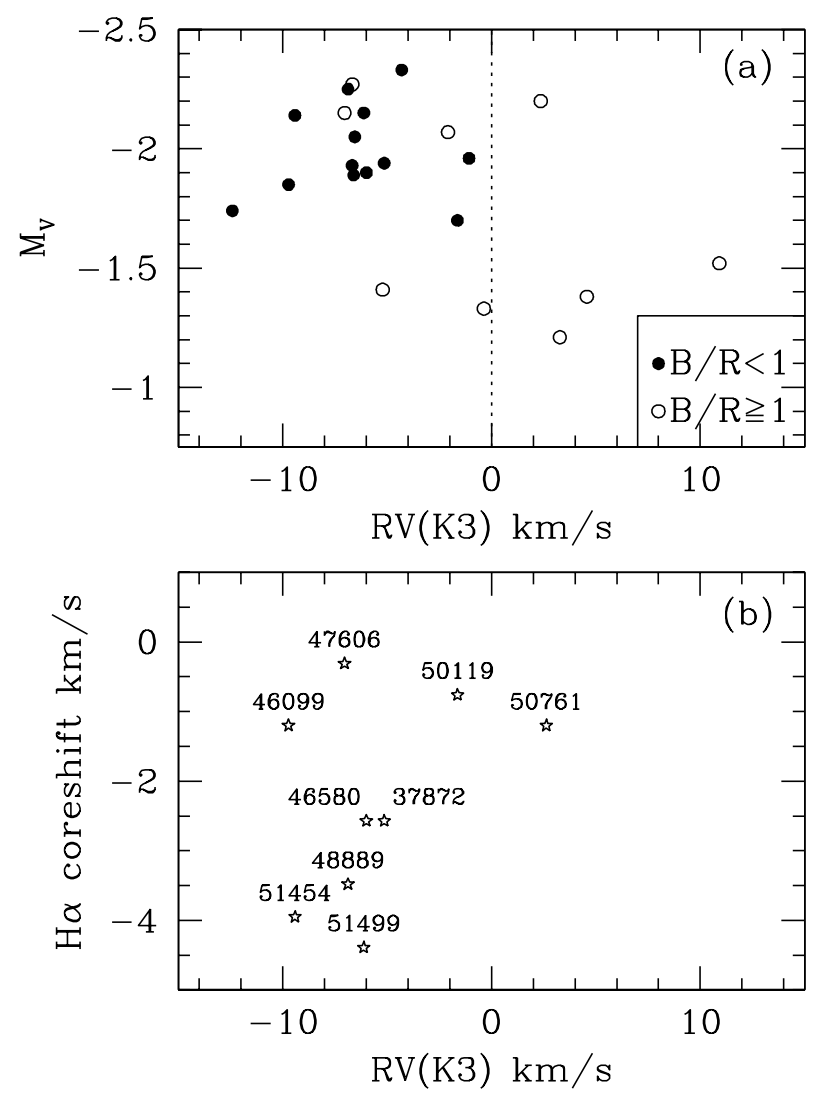

Fig. 10. Panel a): $K_{3}$ velocity shifts as a function of luminosity. Stars with red asymmetry in the $K_{2}$ components (i.e. $B / R<1$ ) are shown as filled circles, stars with blue asymmetry (i.e. $B / R>1$ ) are indicated as open circles. Panel b): $\mathrm{H} \alpha$ coreshift vs. $K_{3}$ velocity shifts for the 9 stars that have both sets of measures.

shifts as a function of $M_{V}$ magnitude, where stars with red and blue $K_{2}$ emission asymmetries are indicated with different symbols: as expected, negative $K_{3}$ velocity shifts are more often associated with red asymmetries, reinforcing the indication of outward motions. These features are also found, to a much larger extent, in Population I bright giants where they suggest the presence of strong cool winds (cf. Dupree 1986). In Population II giants these winds, if present, would appear to be much weaker based on these diagnostics. In Fig. 10b we show the behaviour of the $\mathrm{H} \alpha$ coreshifts relative to the $K_{3}$ velocity shifts for the 9 stars that have both sets of measures: 7 out of 9 stars define a clear tendency for more negative $\mathrm{H} \alpha$ coreshifts to be associated with more negative $K_{3}$ coreshifts, stressing again the indication of outward motion; two stars (46099 and 47606) fall out of this trend, and this could be due to observational errors or variability in the $\mathrm{H} \alpha$ profile, without pretending however to overinterpret the data.

Among our sample stars, the onset of negative $K_{3}$ coreshifts occurs at $\log L / L_{\odot} \sim 2.8$, i.e. at a slightly lower luminosity than the onset of red asymmetry, and it applies to about $89 \%$ of the stars brighter than this value. This threshold is $\sim 0.2 \mathrm{mag}$ fainter than the threshold estimated by Dupree \& Smith (1995) and Smith \& Dupree (1992) from metal-poor field red giants, who find significant negative $K_{3}$ shifts only among stars more 
luminous than $M_{V}=-1.7$ (i.e. $\log L / L_{\odot} \sim 2.88$ ), in a proportion of $\sim 73 \%$.

\subsubsection{The Wilson-Bappu effect}

The full width $W$ of the emission reversal has been shown to be related to the absolute magnitude $M_{V}$ of a red giant stars (Wilson \& Bappu 1957). The most recent calibration based on a sample of 119 nearby stars $\left(M_{V}\right.$ derived from the Hipparcos database and $W\left(K_{2}\right)$ from high resolution spectra) has been provided by Pace et al. (2003), where an extensive discussion and references to the many previous studies are also given. The most recent analogous survey of metal-poor giants was presented by Dupree \& Smith (1995), who analysed 24 metalpoor field red giants and found that, on average, they are more luminous than Population I giants at a given value of the Wilson-Bappu width $W$.

We have measured $W$ for 22 of our stars following the same criteria described by Dupree \& Smith (1995) and Pace et al. (2003), and we list the values of $\log W$ in Table 5. We then show in Fig. 11 the values of $\log W$ as a function of $M_{V}$, and compare them with the calibration for Population I stars given by Pace et al. (2003). In very close agreement with Dupree \& Smith (1995) we also find that the luminosity distribution of our Population II giants is nearly flat and mostly contained within the interval $\left\langle M_{V}>\sim-1.9 \pm 0.3 \mathrm{mag}\right.$, falling above the bright end of the $M_{V}-\log W$ distribution for Population I giants.

In more detail, if we consider only the 17 stars brighter than $V=14$, they have $\langle V\rangle=13.55$ and $\log \langle W\rangle=$ 1.948: if we enter these values in the Pop. I relation we find $<M_{V}>=-1.86$ and $(m-M)_{V}=15.41$. These estimates compare very well with $\left\langle M_{V}\right\rangle=-2.04$ based on the assumed distance modulus $(m-M)_{V}=15.59$, given the accuracy of this method on absolute magnitude (hence distance) determinations. This implies that, at least for these bright giants up to the metallicity of NGC $2808([\mathrm{Fe} / \mathrm{H}] \sim-1.2)$, the dependence of the Wilson-Bappu law on metallicity seems smaller than estimated by Dupree \& Smith (1995). Their statement "Use of the observed $\mathrm{Ca}$ II $\mathrm{K}$ line width to derive an absolute magnitude with a calibration based on Population I stars will underestimate the true $<M_{V}>$ of a metal-deficient giant" is still true, but the error does not seem dramatic.

\section{Summary and conclusions}

We have observed a total of 137 RGB stars in the globular cluster NGC 2808 with the multi-fibre spectrograph FLAMES, and have measured the $\mathrm{Ca}$ II $\mathrm{K}, \mathrm{Na}$ I D and $\mathrm{H} \alpha$ lines for 83, 98 and 98 stars respectively. We have searched for evidence of mass motions in the atmospheres using diagnostics such as absorption line coreshifts and asymmetries in emission components. This is the first time that such a large sample of globular cluster RGB stars have been observed in all the major optical lines that are normally used to study the presence of chromospheres and/or mass motions in the atmospheres.

Four of these stars are not cluster members, as their radial velocities differ by more than $3 \sigma$ from the cluster average

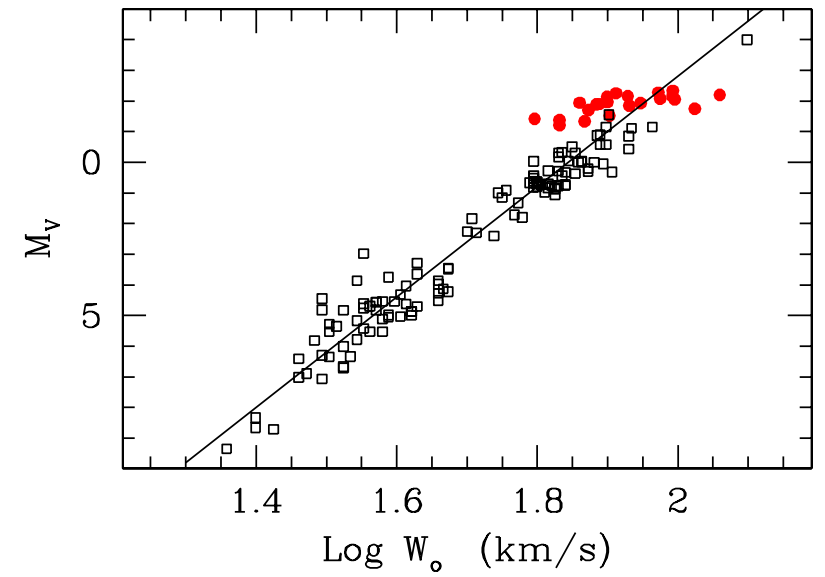

Fig. 11. Wilson-Bappu effect: $\log W$ as a function of $M_{V}$. The open squares show the Population I giants, and the filled circles show the NGC 2808 giants listed in Table 5. The line is the relation for Pop. I giants derived by Pace et al. (2003), $M_{V}=33.2-18 \log W$ (rms error of the fitting is $0.6 \mathrm{mag}$ ).

velocity; for another star the metal abundance is abnormally large, suggesting either that the star is non-member or that it is an AGB star (hence the physical parameters used to calculate the metal abundance are incorrect).

From the remaining sample, we have obtained the following results:

- After subtracting a supposedly pure $\mathrm{H} \alpha$ absorption template profile, we detect evidence of $\mathrm{H} \alpha$ emission down to $\log L / L_{\odot} \sim 2.5$ for $\sim 72 \%$ of stars; this proportion increases with luminosity and becomes $~ 94 \%$ ( 95\%) among stars brighter than $\log L / L_{\odot}=2.7$ (2.9). Compared to all previous studies, including those based on higher spectral resolution such as Lyons et al. (1996), we have set the detection threshold for $\mathrm{H} \alpha$ emission to a fainter limit and increased the fraction of stars where emission could be detected.

The nature of this emission is not assessed definitely yet: our data favour a chromospheric rather than a circumstellar origin, however $\mathrm{H} \alpha$ emission may be present in either stationary or moving chromospheres. Line coreshifts and asymmetries should in principle help distinguish between these two possibilities. For the coreshifts we have used only the 20 stars observed with UVES because of the better spectral resolution. We find that 7 out of these 20 stars, all brighter than $\log L / L_{\odot} \sim 2.88$, show significant $\mathrm{H} \alpha$ coreshifts. In all cases the shifts are less than $9 \mathrm{~km} \mathrm{~s}^{-1}$, and negative (i.e. indicative of outward motion in the layer of the atmosphere where the $\mathrm{H} \alpha$ line is formed).

Asymmetry of the $\mathrm{H} \alpha$ emission wings, indicated as the ratio $B / R$ of the blue and red wing intensities, is mostly blue and does not seem to be correlated with the absorption coreshifts. However, its interpretation is likely complex as it is well known from previous studies that both the intensity of the emission wings and the sense of the asymmetry can be variable with time.

- $\mathrm{Na} \mathrm{D}_{1}$ and $\mathrm{D}_{2}$ lines were observed for 20 stars with UVES and 82 stars with GIRAFFE. $\mathrm{D}_{2}$ line coreshifts were 
measured for all of them, but significant negative values (i.e. $\geq 3 \sigma$ ) were found only among UVES stars brighter than $\log L / L_{\odot} \sim 2.9$, with a detection frequency of about $73 \%$. This luminosity threshold for the onset of significant $\mathrm{Na} \mathrm{D}_{2}$ negative coreshifts is the same as the value found by Lyons et al. (1996), whose detection frequency is however of $\sim 50 \%$. The values we find for the $\mathrm{D}_{2}$ coreshifts reach at most $-4 \mathrm{~km} \mathrm{~s}^{-1}$, whereas Lyons et al. (1996) find values up to $-8 \mathrm{~km} \mathrm{~s}^{-1}$; this might be a real effect, or might be at least partly due to the lower resolution of our data.

Equivalent widths of the $\mathrm{Na}$ I $\mathrm{D}$ lines have been derived for all stars, and used in a separate paper (Carretta et al. 2003) to perform a detailed abundance analysis. Because of possible intrinsic variations of $\mathrm{Na}$ abundance among RGB stars in a given cluster at any luminosity level, a significant fraction of negative $\mathrm{D}_{2}$ line coreshifts might escape detection if the corresponding $E W\left(\mathrm{D}_{2}\right)$ is smaller than the detec-

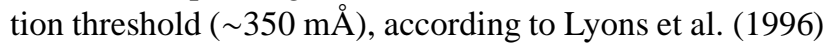
results.

- We have observed with GIRAFFE the Ca II $\mathrm{K}$ line for 83 stars, 22 of which show the central emission $K_{2}$ and reversal $\mathrm{K}_{3}$ features. The detection threshold for these features is $\log L / L_{\odot} \sim 2.6$, and involves about $50 \%$ of our observed sample in this upper luminosity interval.

Asymmetry $B / R$ (i.e. the intensity ratio of the $\mathrm{K}_{2 \mathrm{~b}}$ and $\mathrm{K}_{2 \mathrm{r}}$ components) could be detected in about $75 \%$ of stars brighter than $\log L / L_{\odot} \sim 2.9$, and is mostly $\operatorname{red}(B / R<$ 1) indicating outward motion.

Velocity shifts of the $\mathrm{K}_{3}$ reversal relative to the photospheric LSR have been measured, and are mostly negative indicating that there is an outflow of material in the region of formation of the $\mathrm{K}_{3}$ core reversal. The onset of negative $K_{3}$ coreshifts occurs at $\log L / L_{\odot} \sim 2.8$, i.e. at a slightly lower luminosity level than the onset of red asymmetry, and it applies to nearly $90 \%$ of the stars brighter than this value. Compared to previous results on metal-poor field red giants by Dupree \& Smith (1995) and Smith \& Dupree (1992), we have moved the detection threshold of negative $\mathrm{K}_{3}$ coreshifts about 0.2 mag fainter, and we have increased by more than $20 \%$ the fraction of stars that display such property.

- We have measured the full width $W$ of the Ca II $K_{2}$ emission reversal in the same 22 stars discussed above, and compared them with the Wilson-Bappu relation, $\log W$ as a function of $M_{V}$, defined by Pace et al. (2003) for Population I giants. We find that our Population II giants have a nearly constant luminosity $\left\langle M_{V}\right\rangle \sim-1.9 \pm$ $0.3 \mathrm{mag}$, slightly brighter than the $M_{V}-\log W$ distribution for Population I giants, confirming the results by Dupree \& Smith (1995). Using the 17 brightest stars of our sample, we find that the dependence of the Wilson-Bappu law on metallicity is smaller than previously estimated.

In conclusion, our survey of RGB stars in NGC 2808 searching for mass motion diagnostics in their atmospheres has been able to reach fainter luminosity thresholds and monitor in much more detail along the RGB than any previous study in a given globular cluster. This is due to the FLAMES ability of reaching faint magnitudes with good $S / N$ and good spectral resolution for a large number of stars simultaneously.

Although some of our diagnostics (e.g. $\mathrm{H} \alpha$ emission) may not provide an unambiguous interpretation, other diagnostics give clear indications of the presence of both chromospheres and mass outflows in the atmospheres of these stars. However, we did not attempt to derive any estimate of mass loss rate, that depends on rather uncertain parameterizations of this phenomenon.

Acknowledgements. We are indebted to G. Piotto for the photometry and astrometry of our targets, and to T. Kinman for interesting comments about the early spectroscopic work on NGC 2808. We thank the referee (A. K. Dupree) for useful comments and suggestions. We thank the ESO staff (in particular F. Primas) for carrying out the observations and the preliminary data reduction. This publication makes use of data products from the Two Micron All Sky Survey, which is a joint project of the University of Massachusetts and the Infrared Processing and Analysis Center/California Institute of Technology, funded by the National Aeronautics and Space Administration and the National Science Foundation.

\section{References}

Alonso, A., Arribas, S., \& Martinez-Roger, C. 1999, A\&AS, 140, 261

Alonso, A., Arribas, S., \& Martinez-Roger, C. 2001, A\&A, 376, 1039 (Erratum)

Bates, B., Catney, M. G., \& Keenan, F. P. 1990, MNRAS, 245, 238

Bates, B., Kemp, S. N., \& Montgomery, A. S. 1993, A\&AS, 97, 937

Cacciari, C., \& Freeman, K. C. 1983, ApJ, 268, 185

Cardelli, J. A., Clayton, G. C., \& Mathis, J. S. 1989, ApJ, 345, 245

Carpenter, J. M. 2001, AJ, 121, 2851

Carretta, E., Bragaglia, A., Cacciari, C., \& Rossetti, E. 2003, A\&A, 410,143

Castellani, V., \& Renzini, A. 1968, Ap. Space Sci., 2, 310

Christy, R. F. 1966, ApJ, 144, 108

Cohen, J. G. 1976, ApJ, 203, L127

Cohen, J. G. 1978, ApJ, 223, 487

Cohen, J. G. 1979, ApJ, 231, 751

Cohen, J. G. 1980, ApJ, 241, 981

Cohen, J. G. 1981, ApJ, 247, 869

D’Cruz, N. L., Dorman, B., Rood, R. T., \& O'Connell, R. W. 1996, ApJ, 466, 359

Dupree, A. K., Hartmann, L., \& Avrett, E. H. 1984, ApJ, 281, L37

Dupree, A. K., Sasselov, D. D., \& Lester, J. B. 1992, ApJ, 387, L85

Dupree, A. K., Hartmann, L., Smith, G. H., et al. 1994, ApJ, 421, 542

Dupree, A. K., \& Smith, G. H. 1995, AJ, 110, 405

Faulkner, D. J., \& Smith, G. H. 1991, ApJ, 380, 441

Freire, P. C., Kramer, M., Lyne, A. G., et al. 2001, ApJ, 557, L105

Fusi Pecci, F., \& Renzini, A. 1975, A\&A, 39, 413

Fusi Pecci, F., \& Renzini, A. 1976, A\&A, 46, 447

Fusi Pecci, F., Ferraro, F. R., Bellazzini, M., et al. 1993, AJ, 105, 1145

Gratton, R. G., Pilachowski, C. A., \& Sneden, C. 1984, A\&A, 132, 11

Gratton, R. G. 1988, Rome Obs. Preprint Ser., 29

Harris, W. E. 1996, AJ, 112, 1487 (2003 update in http://physun.physics.mcmaster.ca/Globular.html)

Hesser, J. E., Shawl, S. J., \& Meyer, J. E. 1986, PASP, 98, 403

Iben, I., \& Rood, R. T. 1970, ApJ, 161, 587

Kinman, T. D. 1959, MNRAS, 119, 157

Lyons, M. A., Kemp, S. N., Bates, B., \& Shaw, C. R. 1996, MNRAS, 280,835

Mallia, E. A., \& Pagel, B. E. 1978, MNRAS, 184, 55P 
Montegriffo, P., Ferraro, F. R., Origlia, L., \& Fusi Pecci, F. 1998, MNRAS, 297, 872

Mulas, G., Modigliani, A., Porceddu, I., \& Damiani, F. 2002, Automatic data reduction in support of the FLAMES-UVES VLT Facility, 2002, Proc. of SPIE, 4844, 310

Origlia, L., Ferraro, F. R., Fusi Pecci, F., \& Rood, R. T. 2002, ApJ, 571,458

Pace, G., Pasquini, L., \& Ortolani, S. 2003, A\&A, 401, 997

Pasquini, L., \& Pallavicini, R. 1991, A\&A, 251, 199

Pasquini, L., Avila, G., Blecha, A., et al. 2002, The Messenger, 110, 1

Peterson, R. C. 1981, ApJ, 248, L31

Peterson, R. C. 1982, ApJ, 258, 499

Piotto, G., et al. 2003, in preparation

Reimers, D. 1975, Mem. Soc. R. Sci. Liège, 6th Ser. 8, 369

Reimers, D. 1977a, A\&A, 57, 395
Reimers, D. 1977b, A\&A, 61, 217

Renzini, A. 1977, in Advanced Stages of Stellar Evolution, ed. P. Bouvier, \& A. Maeder, Saas-Fee (Geneva Observatory), 149

Roberts, M. S. 1988, in Globular Cluster Systems in Galaxies, ed. J. E. Grindlay, \& A. G. D. Philip (Dordrecht: Reidel), 411

Rood, R. T. 1973, ApJ, 184, 815

Rutledge, G. A., Hesser, J. E., Stetson, P. B., et al. 1997, PASP, 109, 883

Smith, G. H., \& Dupree, A. K. 1988, AJ, 95, 1547

Smith, G. H., Wood, P. R., Faulkner, D. J., \& Wright, A. E. 1990, ApJ, 353,168

Smith, G. H., Dupree, A. K., \& Churchill, C. W. 1992, AJ, 104, 2005

Walker, A. R. 1999, AJ, 118, 432

Webbink, R. F. 1981, ApJS, 45, 259

Wilson, O. C., \& Bappu, M. K. V. 1957, ApJ, 125, 661 